J. GONZALEZ-TREJO*, C.A. REAL-RAMIREZ*\#, R. MIRANDA-TELLO**, F. RIVERA-PEREZ*,

F. CERVANTES-DE-LA-TORRE*

\title{
NUMERICAL AND PHYSICAL PARAMETRIC ANALYSIS OF A SEN WITH FLOW CONDITIONERS IN SLAB CONTINUOUS CASTING MOLD
}

\begin{abstract}
Some of the most recent technologies that improves the performance in continuous casting process has installed infrastructure outside the mold to modify the natural fluid flow pattern to obtain a quasi-steady condition and promote a uniform solidified shell of steel. The submerged entry nozzle distributes the liquid steel in the mold and can be used to obtain the flow symmetry condition with external geometry improvements. The fluid flow conditioners were located near the outlet ports of the nozzle. The aim of the modifiers is to impose a pseudo symmetric pattern in the upper zone of the mold by inhibiting the fluid exchange between the zones created by conditioners. This work evaluates the effect of the thickness and length of the fluid-flow modifiers on the overall performance of the submerged nozzle. These properties of the fluid-flow modifiers were normalized based on two of the geometric dimensions of the standard equipment. Numerical and physical simulations suggest that the flow modifier should be as thin as possible.
\end{abstract}

Keywords: Submerged entry nozzle, fluid-flow modifiers, continuous casting of steel, mold flow patterns, turbulence

\section{Introduction}

For several years, the continuous casting of steel slabs has been considered a mature technology. However, several researchers from both industry and academia have proven various devices and operating strategies in order to improve the quality of steel produced through this process [1-4]. Surface defects and slag entrapment are significant defects that deteriorate the quality of steel that is produced by the continuous casting process [5-10].

It is well known that fluid flow pattern inside the mold that minimizes the number of defects in the steel produced by continuous casting is named as the double roll (DR) [11]. Two other fluid flow patterns inside the mold can be observed depending on the operating conditions and the characteristics of casting equipment. These flow patterns are the single roll (SR) and the unstable pattern (UP). These two patterns are undesirable because of the problems caused in the semi-finished products. Recently, some studies have reported mappings of the operating conditions that generate each of these three main types of fluid flow patterns inside the mold [11,12].

Researchers have used several strategies in isolation or combined to remove or at least reduce the number and severity of the internal and external defects of solidified steel. Directly or indirectly, these strategies are aimed to induce a DR-like fluid flow pattern inside the mold. Some strategies with particular relevance are the following:

a) Changes in operating conditions [11].

b) Changes of the geometric features of the components forming the casting equipment [13].

c) The use of external devices to change the fluid flow pattern inside the casting equipment, such as the electromagnetic brake $[14,15]$.

Immersing external refractory shapes in the mold is another approach recently proposed by Kamal and Sahai [14]. The authors claimed that their device was a cheaper and more efficient solution to the problem.

Following Kamal and Sahai, Rivera-Perez et al. [16] presented a SEN with fluid flow modifiers attached to the external wall of a standard bifurcated SEN. The flow conditioners impose a pseudo-symmetric pattern in the upper zone of the mold by inhibiting the fluid exchange between the zones created by conditioners. The hypothesis behind the design is that fluid flow conditioners will reduce the oscillations of the molten steel level and, for this reason, the occurrence of vortexes at the interface between slag and molten steel is also reduced. The vortexes at this zone are harmful because they can entrap clusters of the slag floating above the molten steel and send it to the semi-finished product.

\footnotetext{
* SYSTEMS DEPARTMENT, UNIVERSIDAD AUTONOMA METROPOLITANA-AZCAPOTZALCO, AV. SAN PABLO 180, REYNOSA-TAMAULIPAS, AZCAPOTZALCO, 02200, MEXICO CITY, MEXICO ** ELECTRONICS DEPARTMENT, UNIVERSIDAD AUTONOMA METROPOLITANA - AZCAPOTZALCO, AV. SAN PABLO 180, REYNOSA-TAMAULIPAS, AZCAPOTZALCO, 02200, MEXICO CITY, MEXICO

\# Corresponding author: carr@azc.uam.mx
} 
The reduction of the slag entrapment is the main objective of the proposed device. The entrapment is produced by fluid streams that cross the mold perpendicularly from the axis of the jet. These streams can be detected as variations in the steel liquid level. Therefore, the performance of the device would be better as the fluid flow pattern at the mold upper section and the liquid level be as stable as possible.

The variations in the fluid flow pattern inside the SEN can be partially controlled by manipulating the stopper at the tundish section nevertheless, the characteristics and the shape of the jets ejected from the SEN exit ports remain the principal source of the variations observed in the steel liquid level inside the mold.

The fluid flow modifiers is a passive technology that aims to absorb the fluctuations of the jets ejecting from the SEN in a similar way as the electromagnetic brakes. The non-consumptions energy feature of the proposed technology eventually could reduce significantly the production costs.

This work evaluates the effect of the thickness and length of the fluid flow modifier on the overall performance of the SEN through numerical and physical simulations. These properties of the modifiers were normalized based on two of the geometric dimensions of the standard equipment, the thickness mold thick and the diameter of the SEN outlet ports.

Rivera-Perez et al. [16] used biphasic numerical simulations in order to show that fluid flow modifiers indeed minimizes the occurrence of vortexes at the liquid free surface. Their numerical simulations used water and air as working fluids. The authors used those fluids because numerical simulations were compared with the results of physical experiments conducted on a scaledmold model with water as working fluid. The purpose of this work is to reconstruct by means of numerical simulations the fluid flow pattern inside the mold generated with different fluid flow modifiers. After that, the properties, implications, advantages and drawbacks of each pattern are discussed. For this reason, all the numerical simulations conducted in this work employed only water as working fluid and using the commercial software ANSYS FLUENT [17]. The heat transfer and the solidification process at the mold section are beyond the scope of this work. However, the effect of the produced fluid flow patterns of both phenomena is briefly discussed.

Numerical results were compared and validated by means of physical simulations conducted on a scaled cold water model, which is a rectangular prism made of acrylic plates. It has a 1:1/3 scale ratio, and its dimensions are $1.200 \mathrm{~m}$ long, $0.500 \mathrm{~m}$ width and $0.090 \mathrm{~m}$ thick. Further details about the physical model can be consulted in [10] and [16].

\section{Description of the proposed SEN with fluid flow modifiers}

The role of the submerged entry nozzle is to distribute liquid steel from the distributor into the mold. The mold is a rectangular shaped jacket, cupper made, which is cooled by recirculation water. For this reason, the liquid molten steel in contact with the walls of the mold abruptly cools and quickly solidifies. This process forms a solidified shell that increases in thickness as it descends in the mold.

Figure 1c is a schematic description of the proposed SEN and the system used for numerical modelling. The main features of a bifurcated SEN with fluid flow conditioners were already reported in [16]. Figure 1a shows a traditional bifurcated SEN. The slab continuous casting mold serves as a visual reference. Figure1b depicts the proposed SEN with fluid flow conditioners. All the fluid flow modifiers studied in this work are solid blocks of rectangular shape. The experimental results presented in [16] showed that the performance of a hollowed fluid flow modifier was also satisfactory. Nevertheless, the characterization of that kind of modifier is out of the scope of this work.

This work evaluates the effect of the geometric dimensions of the fluid flow modifiers by normalizing the size according two of the geometric dimensions of the standard equipment, the thickness of the mold $(w)$ and the diameter of the SEN outlet ports. Figure1b also shows the two parameters analyzed in this work, the thickness of the modifier $(t)$ and its length, this is given indirectly through the gap $(g)$ between the mold narrow wall and the edge of the modifier summarizes the geometrical dimensions of the six fluid flow conditioners studied in this work named as Models A, B, C, D, E, and F. Note that all the fluid flow modifiers investigated in this work has the same height.

Figure 2 shows a schematic representation of the six SEN geometries studied in this work. The thickness of Model A, C and $E$ is nearly a half of the diameter of the inlet ports of the nozzle. Also, this value was chosen because it is almost equal to the thickness of the inner walls of the nozzle. We believe that the thickness ensures the structural integrity of the nozzle in the case of a future implementation at industry. So, the rupture of the nozzle due to the operational conditions which usually operates is a remote event. The thickness of Models B, D, and F is twice the thickness of Models A, C, and E.

For Models $C$ and D, the gap between the edge of the modifier and the narrow wall was reduced to a half of that used in Models A and B. Models E and F are included as limiting cases. In both models, the edges of the flow modifiers reach the narrow walls of the continuous casting mold. This condition is not acceptable for industry since the development of meniscus and would prevent the correct formation of the solidified layer of steel. However, the obtained results with these modifiers would be used to characterize the flow patterns.

\section{Numerical simulation of the SEN}

\subsection{Basic mathematical equations}

If we consider that the working fluid is incompressible, we obtain the following Navier-Stokes equation, which describes the fluid dynamics of a system:

$$
\rho\left(\frac{\partial \vec{v}}{\partial t}+\vec{v} \cdot \nabla \vec{v}\right)=-\nabla p+\eta \nabla^{2} \vec{v}+\rho f
$$



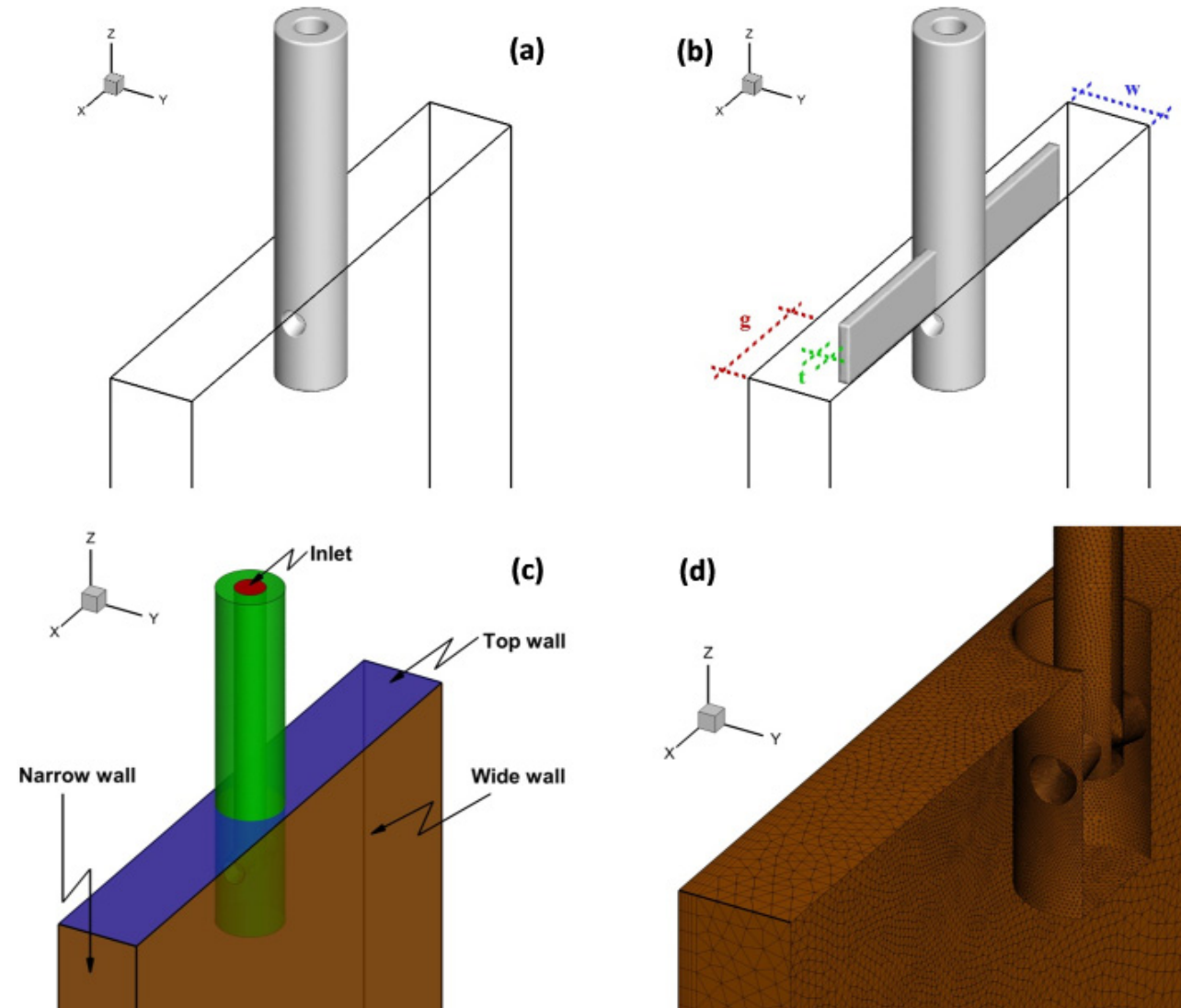

(c)

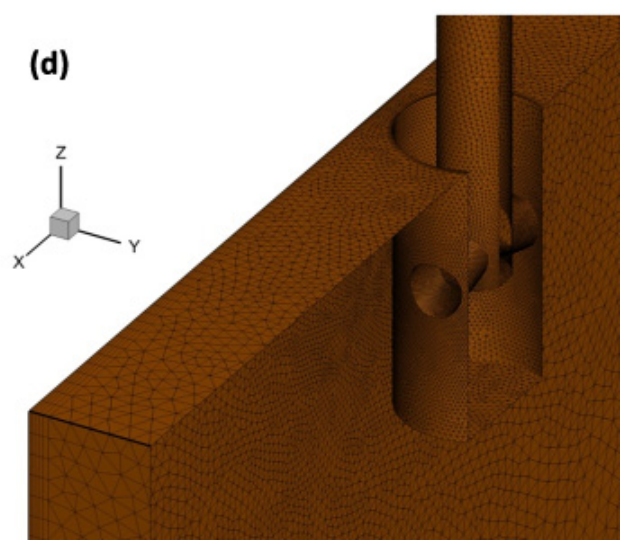

Fig. 1. Schematic description of the system (a) Traditional bifurcated SEN inside the slab continuous casting mold. (b) Proposed SEN with fluid flow conditioners. (c) Description of main components using a standard SEN. (d) Mesh of numerical simulation
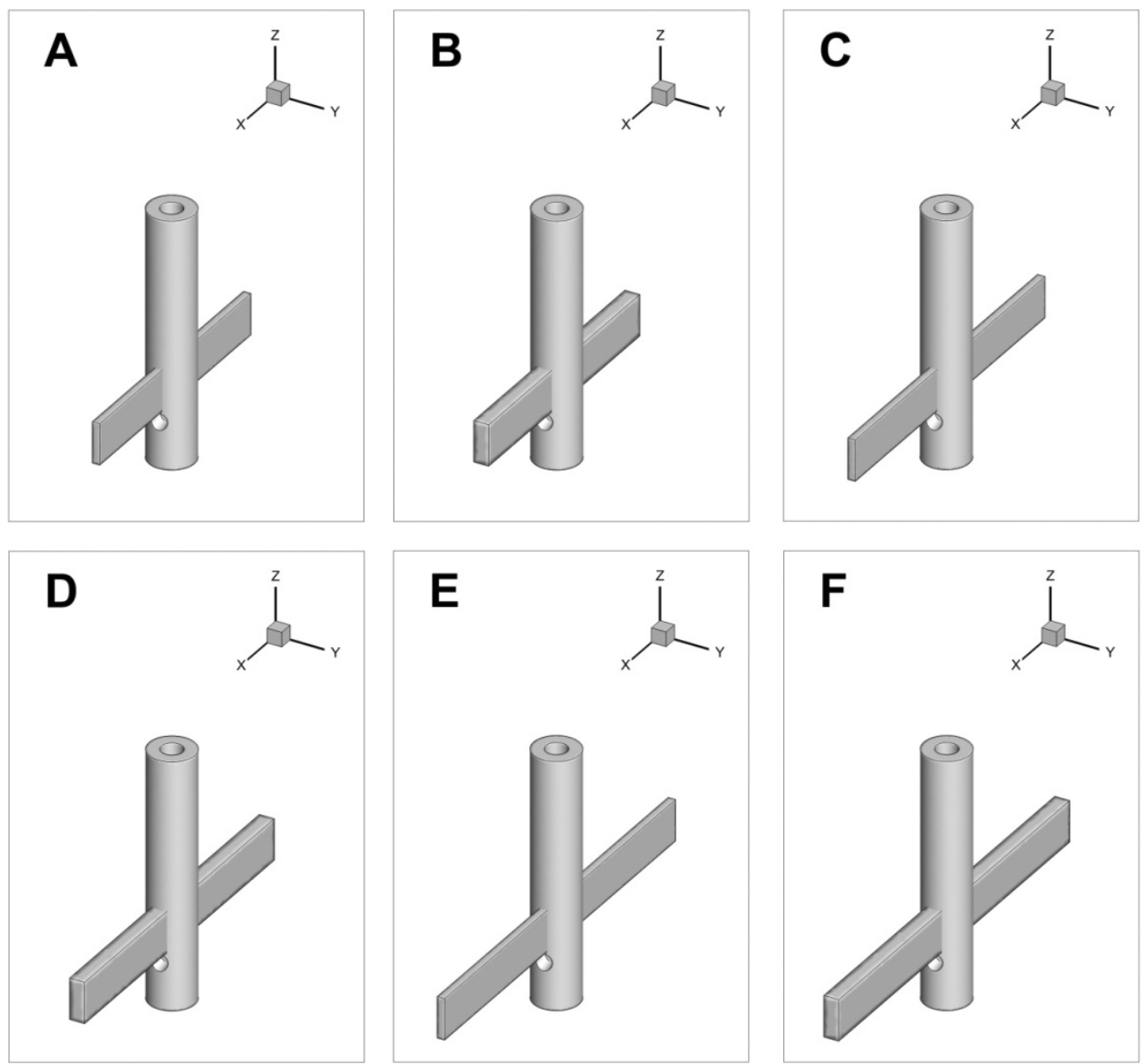

Fig. 2. Schematic representation of the six SEN geometries with fluid flow modifiers studied in this work 
The numerical simulations used the standard $k-\varepsilon$ model for modeling turbulence. The turbulence kinetic energy, $k$, and its rate of dissipation, $\varepsilon$, are obtained from the following transport equations:

$$
\begin{aligned}
\frac{\partial}{\partial t}(\rho k)+\frac{\partial}{\partial x_{i}}\left(\rho k u_{i}\right) & =\frac{\partial}{\partial x_{j}}\left[\left(\mu+\frac{\mu_{t}}{\sigma_{k}}\right) \frac{\partial k}{\partial x_{j}}\right]+ \\
& +G_{K}+G_{b}-\rho \varepsilon-Y_{M}+S_{k}
\end{aligned}
$$

and

$$
\begin{array}{r}
\frac{\partial}{\partial t}(\rho \varepsilon)+\frac{\partial}{\partial x_{i}}\left(\rho \varepsilon u_{i}\right)=\frac{\partial}{\partial x_{j}}\left[\left(\mu+\frac{\mu_{t}}{\sigma_{k}}\right) \frac{\partial \varepsilon}{\partial x_{j}}\right]+ \\
+C_{1 \varepsilon} \frac{\varepsilon}{k}\left(G_{k} C_{3 \varepsilon} G_{b}\right)-C_{2 \varepsilon} \rho \frac{\varepsilon^{2}}{k}+S_{\varepsilon}
\end{array}
$$

In these equations, $G_{K}$ represents the generation of turbulence kinetic energy due to the mean velocity gradients, $G_{b}$ is the generation of turbulence kinetic energy due to buoyancy, $Y_{M}$ represents the contribution of the fluctuating dilatation in compressible turbulence to the overall dissipation rate. $C_{1 \varepsilon}, C_{2 \varepsilon}$ and $C_{3 \varepsilon}$ are constants. $\sigma_{k}$ and $\sigma_{\varepsilon}$ are the turbulent Prandtl numbers for $k$ and $\varepsilon$ respectively. $S_{k}$ and $S_{\varepsilon}$ are the user-defined source terms.

The model constants have the following default values [18]: $C_{1 \varepsilon}=1.4400 ; C_{2 \varepsilon}=1.920 ; C_{3 \varepsilon}=0.090 ; \sigma_{k}=1.000 ; \sigma_{\varepsilon}=1.300$. These values have been determined from experiments for fundamental turbulent flows. Several works have reported that these values are adequate for numerical simulations of the continuous casting of steel.

\subsection{Geometrical dimensions and boundary conditions}

Numerical results of the mathematical model described above will be validated with a scaled model and using water as working fluid. For this reason, the geometrical dimensions and boundary conditions of the mathematical model coincide with the scaled model, which is a rectangular prism made of acrylic plates. It has a 1:1/3 scale ratio, and its dimensions are $1.200 \mathrm{~m}$ long, $0.500 \mathrm{~m}$ width and $0.090 \mathrm{~m}$ thick. The water is discharged into the mold through the SEN, which is a cylindrical bifurcated pipe. The SEN is at the center of the mold, in the upper zone. The dimensions of the SEN are $0.650 \mathrm{~m}$ of length, $0.0557 \mathrm{~m}$ of external diameter and $0.0254 \mathrm{~m}$ of inner diameter. The SEN has two exit ports with a diameter of $0.020 \mathrm{~m}$. Each port has an inclination of 15 degrees downward with respect to the horizontal (see [10] and [16] for further details).

The initial condition for all cases is a fluid at rest, the water level in the mold is $0.150 \mathrm{~m}$ distance to the top of the mold. The SEN submergence depth is equal to $0.050 \mathrm{~m}$, measured from the liquid free surface to the top point of the exit ports. The primary inlet boundary condition is the liquid inlet velocity. Water enters into the mold through the inlet port at the top of the nozzle. The liquid velocity has a constant value of $1.150 \mathrm{~m} \cdot \mathrm{s}^{-1}$. This value corresponds to a nominal casting speed of $1.800 \mathrm{~m} \cdot \mathrm{min}^{-1}$.
The second boundary condition corresponds to the outlet at the bottom of the mold. This wall is as an outflow boundary. The flow rate weighting value is 1.000 .

\subsection{Discretization of numerical models}

The models represent (a) the mold with a traditional SEN and (b) the model using the SEN with fluid flow conditioners, and were discretized employing similar criteria. The size of the minimum element was $1.000 \times 10^{-5} \mathrm{~m}$. Pyramidal elements grow linearly with a constant value of 1.050. Curvature and distance criteria were included. For this reason, models have a bigger elements density in the vicinity of the nozzle and near the liquid free surface.

For illustrative purposes, Figure 1d shows the mesh on the nozzle internal and external walls, as well as the mesh on the top, narrow and wide walls of the mold. This figure includes only a traditional SEN.

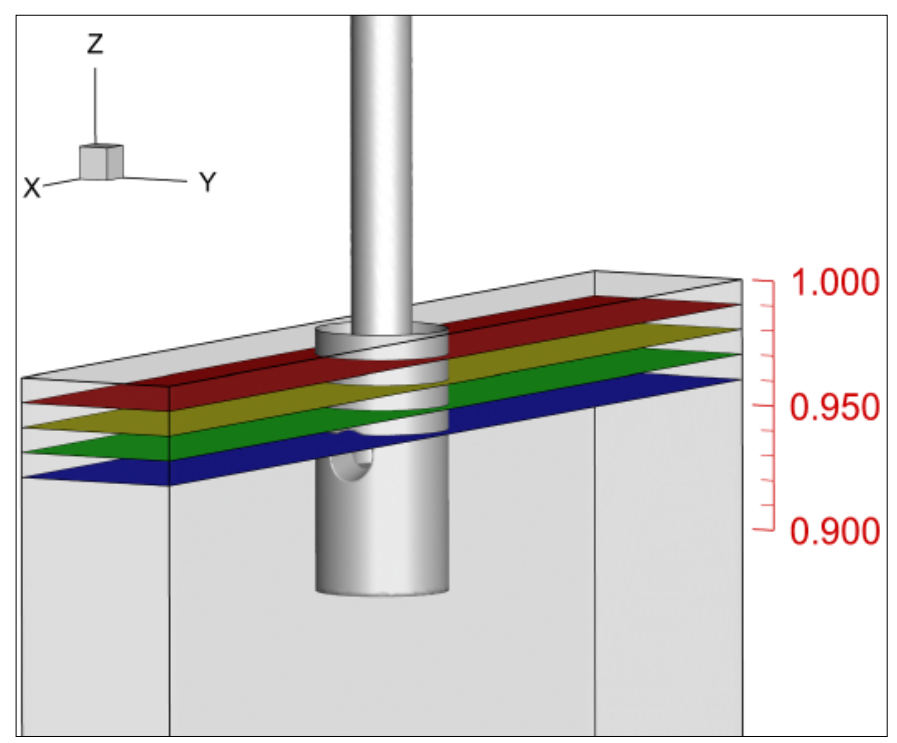

Fig. 3. Spatial location of the four horizontal planes used to visualize the fluid flow pattern near the top of the mold

\subsection{Standard SEN at steady-state condition}

This subsection numerically characterizes the fluid flow pattern inside the continuous casting mold using a standard bifurcated nozzle at steady state conditions. These numerical simulations provide a point of comparison reliable to evaluate the advantages and disadvantages of the proposed SEN geometries. A numerical simulation imposing a steady-state condition average the instantaneous fluctuations of the flow observed in a transient simulation.

Figure 4 shows the shape of the jets emerging from the SEN outlet ports, which are depicted through streamlines. The markers are colored in accordance with its velocity magnitude. Figures $4 \mathrm{e}$ and $4 \mathrm{f}$ display the fluid flow pattern inside the mold, which is depicted through streamlines starting at the SEN inlet 

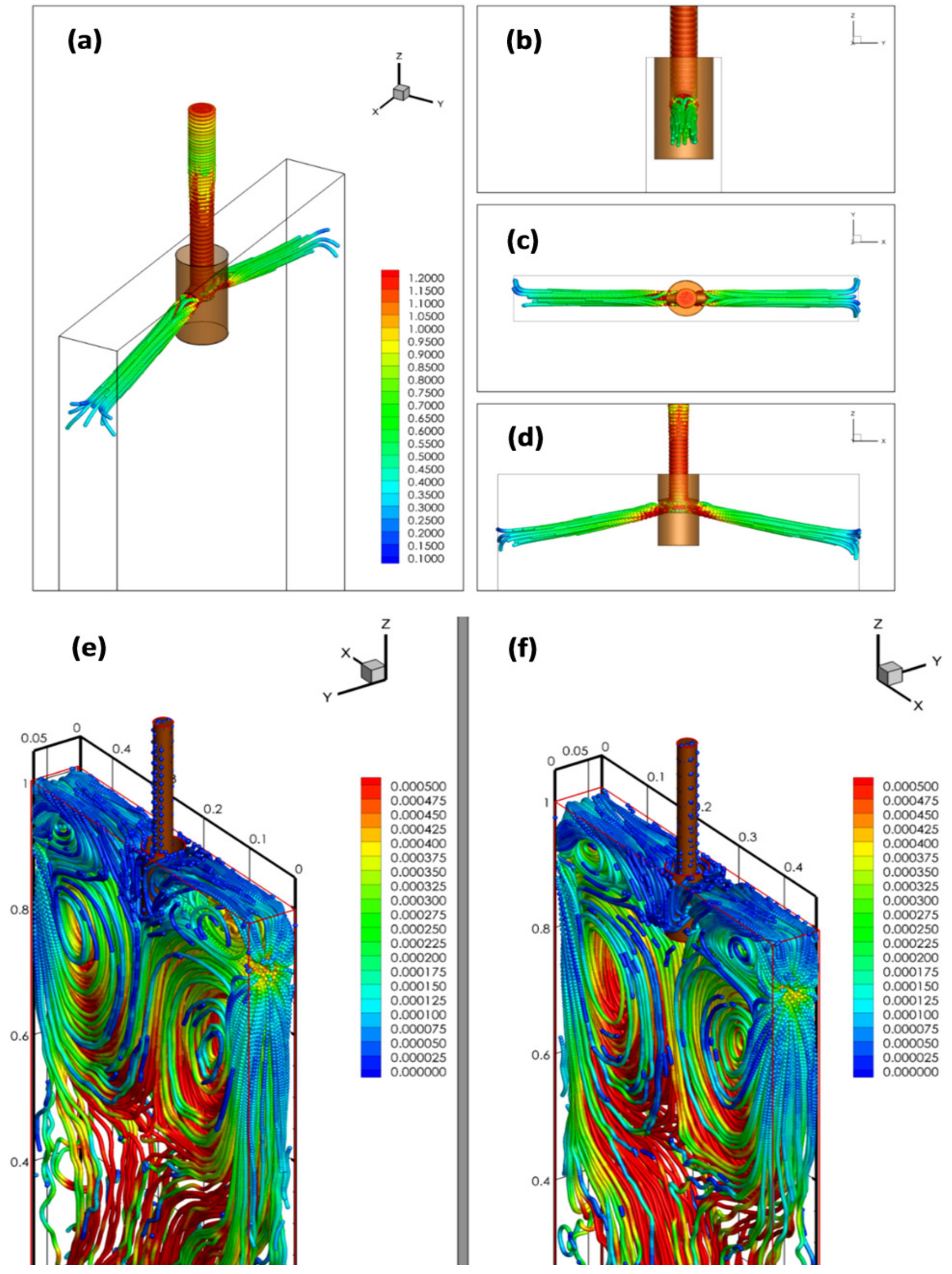

Fig. 4. Numerical simulation of flow pattern at steady condition for a standard SEN, jets emerging from the outlets ports of the SEN, (a) isometric view, (b) lateral view, (c) upper view, (d) front view, units are $\left[\mathrm{m} \cdot \mathrm{s}^{-1}\right.$ ]. Fluid flow pattern inside the mold, (e) isometric view front-wall oriented of mold (f) isometric view back wall oriented of mold, units are $\left[\mathrm{m}^{2} \cdot \mathrm{s}^{-1}\right]$ 
port. In these figures, the streamlines markers are colored in accordance with its turbulent kinematic viscosity magnitude. Both figures show that the fluid flow pattern inside the mold is almost symmetric.

Figure 4 also shows that the fluid jets emerging from the nozzle outlet ports remain narrow and coherent until it reaches the narrow walls of the mold. Both jets nearly match a straight line inclined almost 15 degrees below the horizontal line. This angle coincides with the tilt of the nozzle outlet ports. It is observed that the entire jets impinge against the narrow walls almost at its center.

Figures $4 \mathrm{e}$ and $4 \mathrm{f}$ allow to observe four vortexes or recirculation zones inside the mold at the front and back oriented view. These vortical structures form the double-roll (DR) fluid flow pattern. Note that in these figures, the view shown on the right side is a 180-degree rotation of the view shown on the left side of the figure. For this reason, the figure simultaneously shows the two wide walls and the two narrow walls of the mold. Note that when the fluid jet collides with the narrow wall of the mold, the stream is separated into two parts, one part rises to the top of the mold and the other descends to the bottom of the mold.

Figures $4 \mathrm{e}$ and $4 \mathrm{f}$ also show that in the zone near the top of the mold, the fluid flows predominantly from the narrow wall towards the nozzle. Figure 3 shows the physical location of each of these planes. Note that the four planes are distributed equidistantly in the upper zone of the mold, between the output ports of the nozzle and the top of the mold. Figure 6a shows the velocity vectors at the four horizontal planes in the upper zone of the mold. The height of the plane is indicated on each figure. The plane that crosses the exit ports of the nozzle is shown at the top of the figure while the plane near the upper part of the mold is at the bottom of the figure. Note that the length of the arrows is proportional to the fluid velocity magnitude at that point.

\subsection{Standard SEN under transient conditions}

This subsection reports the transient numerical simulations of a standard bifurcated SEN. The results shown in all the transient simulations were obtained after allowing the process to proceed for 60 seconds. After performing several simulations, we observed that this time was sufficient to remove the onset effect of the numerical simulation. It was also observed that all the models reached a quasi-steady state using 60 seconds of operation time.

As in previous case, Figure 5 shows the shape of the jets emerging from the SEN outlet ports by using streamlines. It is noteworthy that the start points, for constructing the streamlines this figure are the same to that used for the streamlines shown in Figure 4.

Figure 5 shows that the behavior of the fluid jet in transient conditions is different from that observed under steady state conditions. This figure also shows that the behavior of the jet is far from being symmetric in respect the XZ plane. Despite this, the behavior of the jets emerging from the two output ports has a certain degree of symmetry with respect to the YZ plane.

Very soon, the jet's emerging from the outlet ports of the nozzle is scattered. This behavior causes that part of the jet collides with one of the wide walls of the mold and that only a fraction of the jet reaches the narrow wall of the mold. Note that both jets collide with the same wide wall of the mold. This behavior increases the asymmetry of the fluid flow pattern at upper zone of the mold in respect the $\mathrm{XZ}$ plane. This observation is reinforced by analyzing the behavior of the velocity vectors in each of the horizontal planes that were presented in Figure 6b. The results for the standard nozzle under transient conditions are shown in Figures 5 and 6b. Note that Figure 6a and Figure 6b have the same streamlines structure. Figure 5 shows that many streamlines cross the imaginary plane $\mathrm{XZ}$, although the number of crossings decreases as the height of the plane increases.

The fluid flow pattern inside the entire mold is displayed in Figures 5e and 5f. Note that these figures and Figures 4e and $4 \mathrm{f}$ have the same streamlines structure. These figures shows the four vortexes or recirculation zones inside the mold. Note that, in this case, the jets emerging from the outlet ports of the nozzle does not collide with the narrow walls of the mold at its central zone.

\subsection{A nozzle under transient conditions}

Figure 7 shows that in this model, the jets emerging from the outlet ports disperses over a broader area than that observed for the standard nozzle. This figure reveals that a fraction of the jets collides with one of the wide walls of the mold, similar to that observed for the standard nozzle.

The inclusion of fluid flow modifier reduces the volume occupied by the fluid at the top of the mold. As the casting speed is constant, it is natural to expect that the average fluid velocity in this area is increased. This assumption is confirmed by observing the fluid velocity field on each of the horizontal planes showed in Figure 9a (the spatial location of the planes is described in Figure 3). This figure shows that for $\mathrm{Z}$ heights 0.960 $\mathrm{m}$ and $0.970 \mathrm{~m}$, the recirculation zones near the narrow walls of the mold are slightly intense than that observed for the nozzle without fluid flow modifiers. Figure 9a also confirms that the fluid flow modifier fulfills its original purpose. It is observed that for $\mathrm{Z}$ heights $0.980 \mathrm{~m}$ and $0.990 \mathrm{~m}$, almost all of the flow on both sides of the modifier is directed toward the nozzle.

The fluid flow pattern inside the entire mold produced by the Model A nozzle is displayed in Figure 7. Note that these figures and Figures 4 and 5 have the same structure. This figure shows that Model A nozzle produces the four vortexes of the double-roll flow pattern. Comparing Figures 7e and $7 f$ with Figures 5e and 5f, it can be seen that the Model A nozzle produces a fluid flow pattern within the mold that is more symmetrical with respect to the YZ plane. These results confirm the assertions previously published in [16]. 

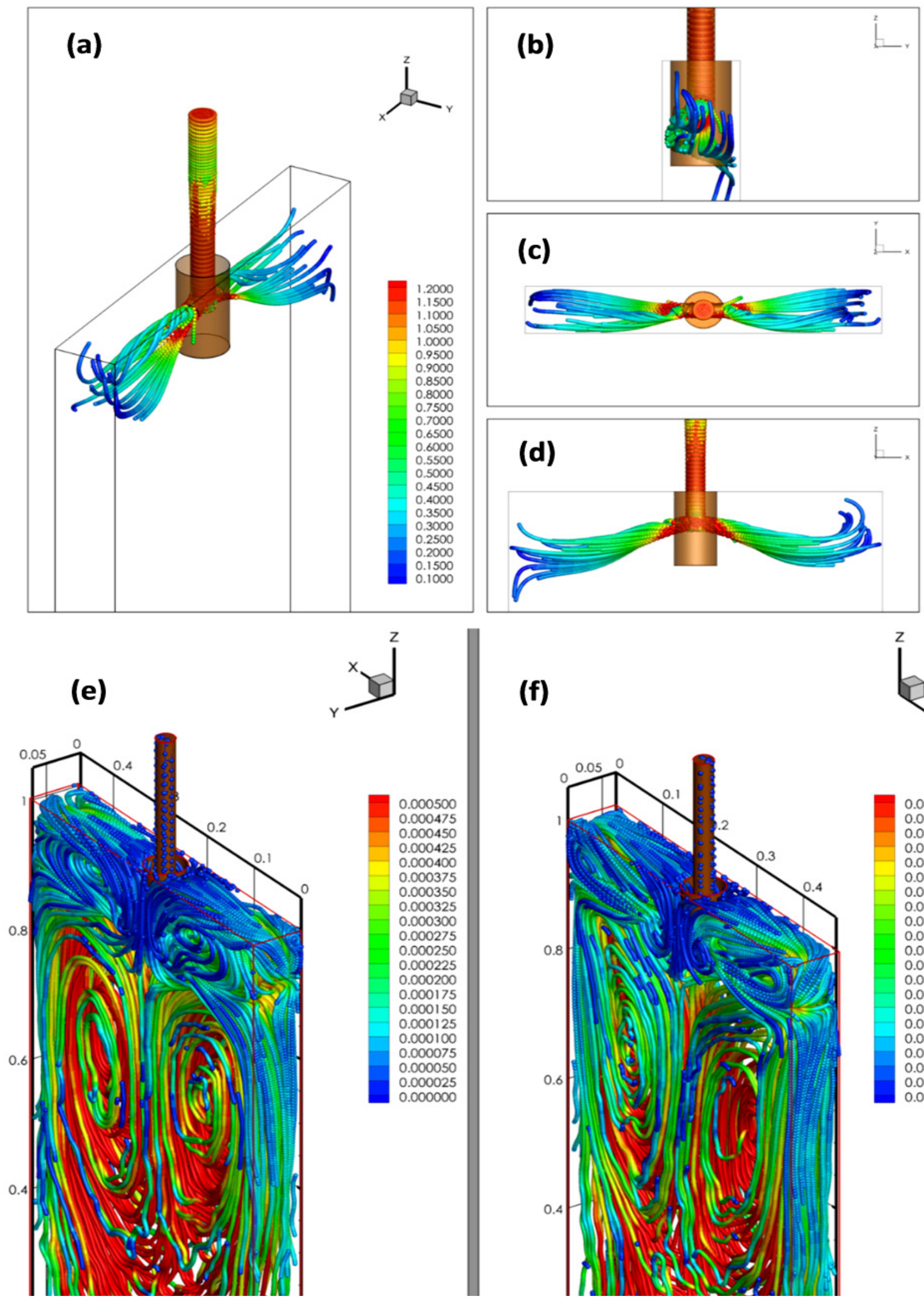

(f)
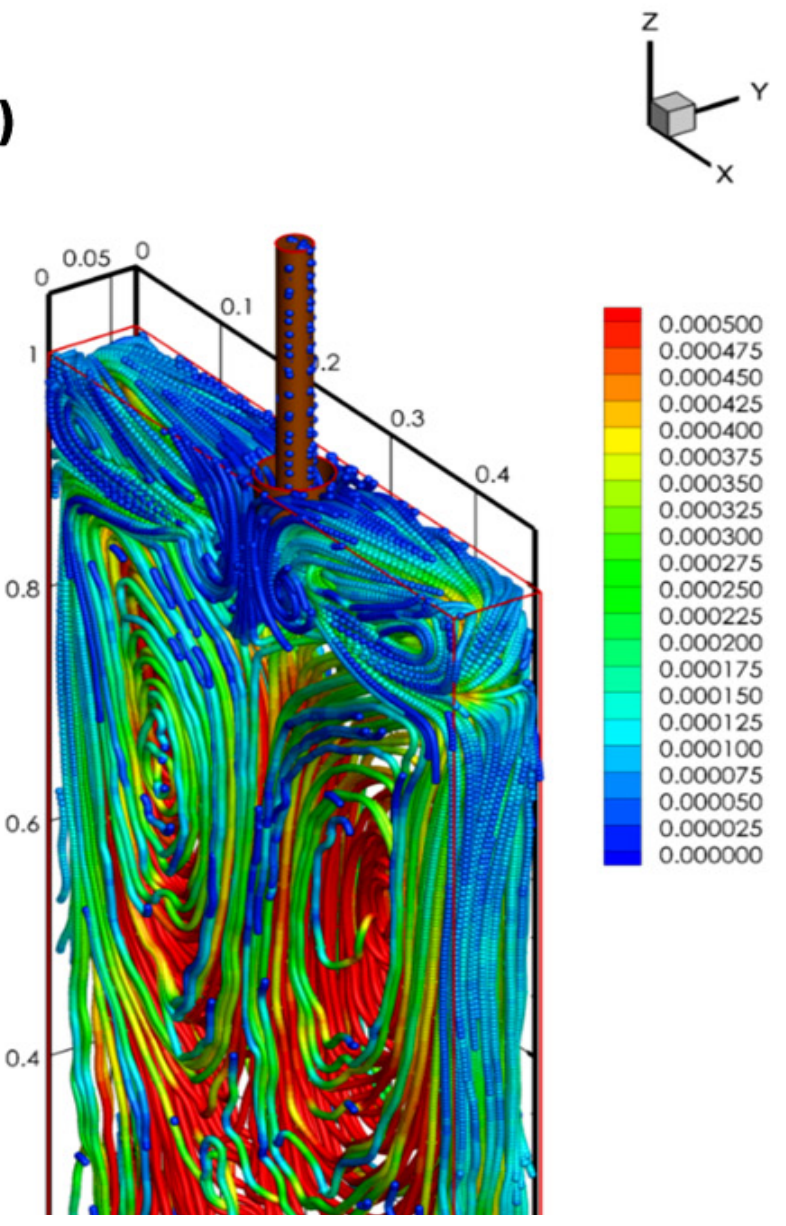

Fig. 5. Numerical simulation of flow pattern under transient condition for a standard SEN, jets emerging from the outlets ports of the SEN, (a) isometric view, (b) lateral view, (c) upper view, (d) front view, units are $\left[\mathrm{m} \cdot \mathrm{s}^{-1}\right]$. Fluid flow pattern inside the mold, (e) isometric view front-wall oriented of mold (f) isometric view back wall oriented of mold, units are $\left[\mathrm{m}^{2} \cdot \mathrm{s}^{-1}\right]$ 


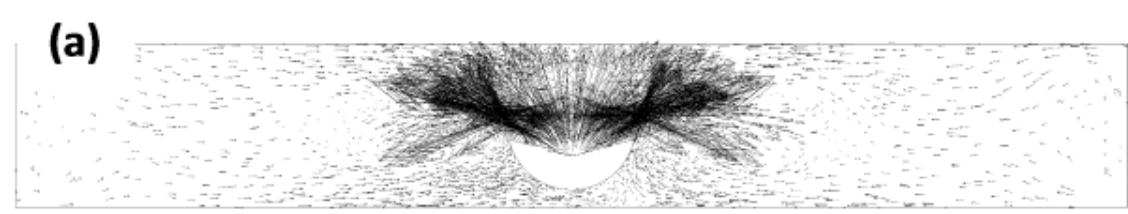

$z=0.960 m$

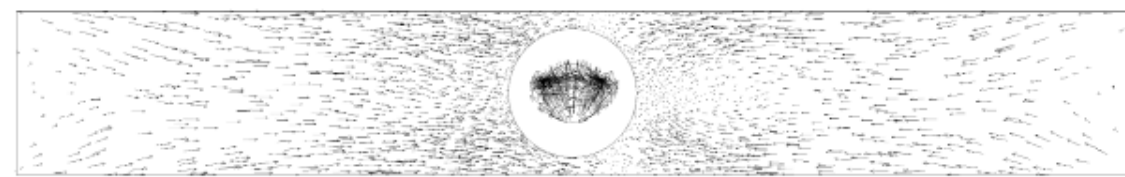

$z=0.970 m$

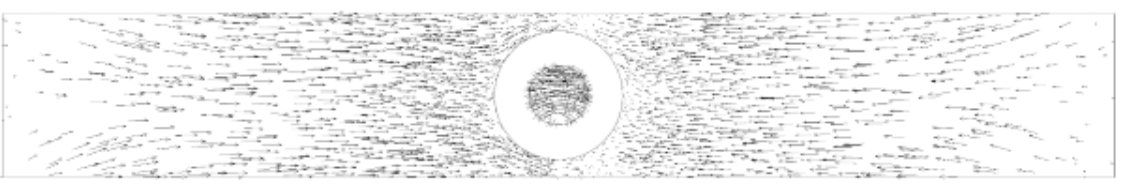

$z=0.980 m$

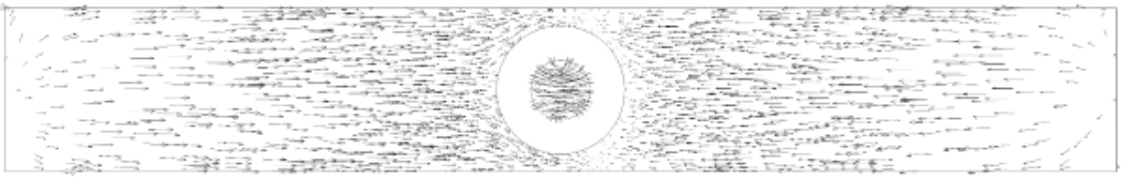

$\mathrm{z}=0.990 \mathrm{~m}$

\section{(b)}

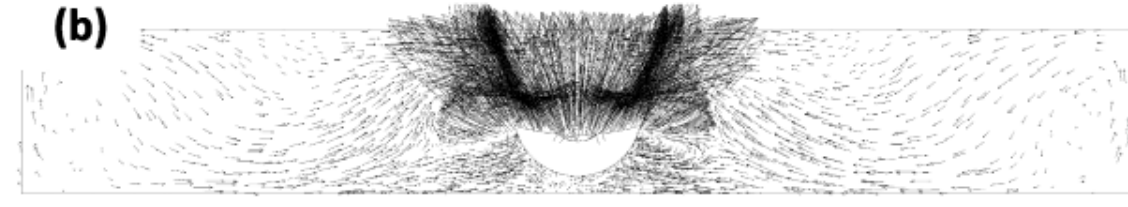

$z=0.960 m$

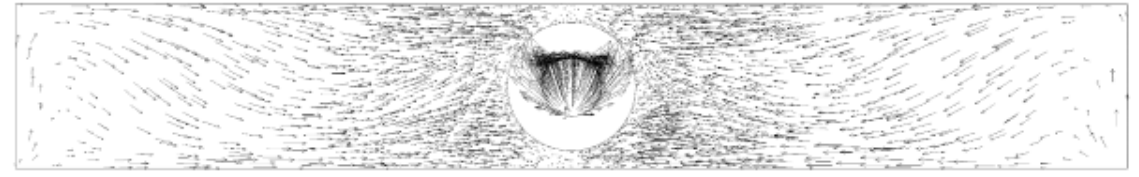

$z=0.970 m$

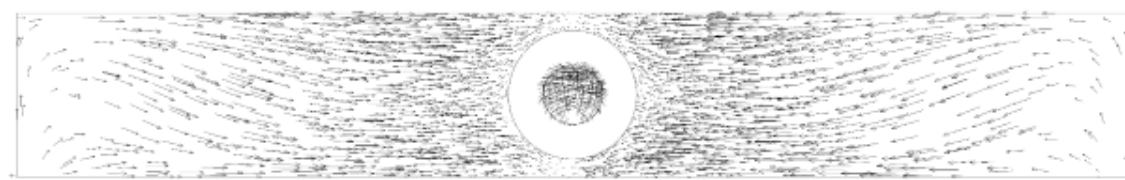

$z=0.980 m$

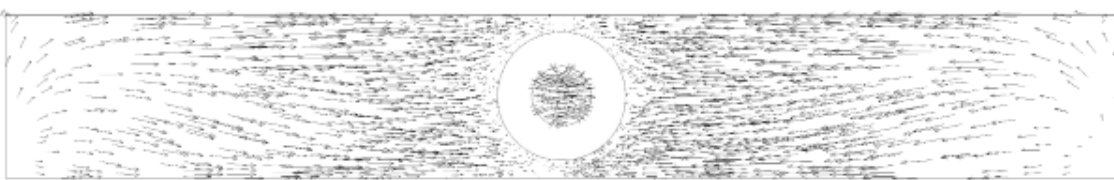

$z=0.990 m$

Fig. 6. Velocity vectors at four horizontal planes in the upper zone of the mold for a standard SEN (a) at steady state condition (b) under transient conditions. The length of the arrows is proportional to the fluid velocity magnitude at that point

\subsection{Model B nozzle under transient conditions}

Figure 8 depicts the shape of the fluid jet produced by Model B nozzle. This figure shows that the volume occupied by the flow modifiers is too large, so the entire jets emerging from the outlet ports of the nozzle directly collide with one of the wide walls of the mold. This behavior is totally unacceptable since it substantially degrades the quality of the solidified layer, which is formed on the mold section.

Figure 9b shows that the performance of the nozzle Model B is inadequate and does not meet the original purpose of flow modifiers. In addition, it can clearly be seen that even near the top of the mold, a large part of the flow on both sides of the modifier has an upward direction. This fluid flow pattern will generate a considerable amount of fluctuations in the level of liquid steel in one of the mold halves. Figures 8 e and $8 \mathrm{f}$ confirm that the Model B nozzle is not a viable option. It can be seen that this nozzle generates into the mold a fluid flow pattern very different to the double-roll (DR) pattern.

\subsection{Model $C$ nozzle under transient conditions}

The shape of the fluid jet produced by Model C nozzle shown in Figure 10 is much more symmetrical than that obtained with a standard nozzle. 

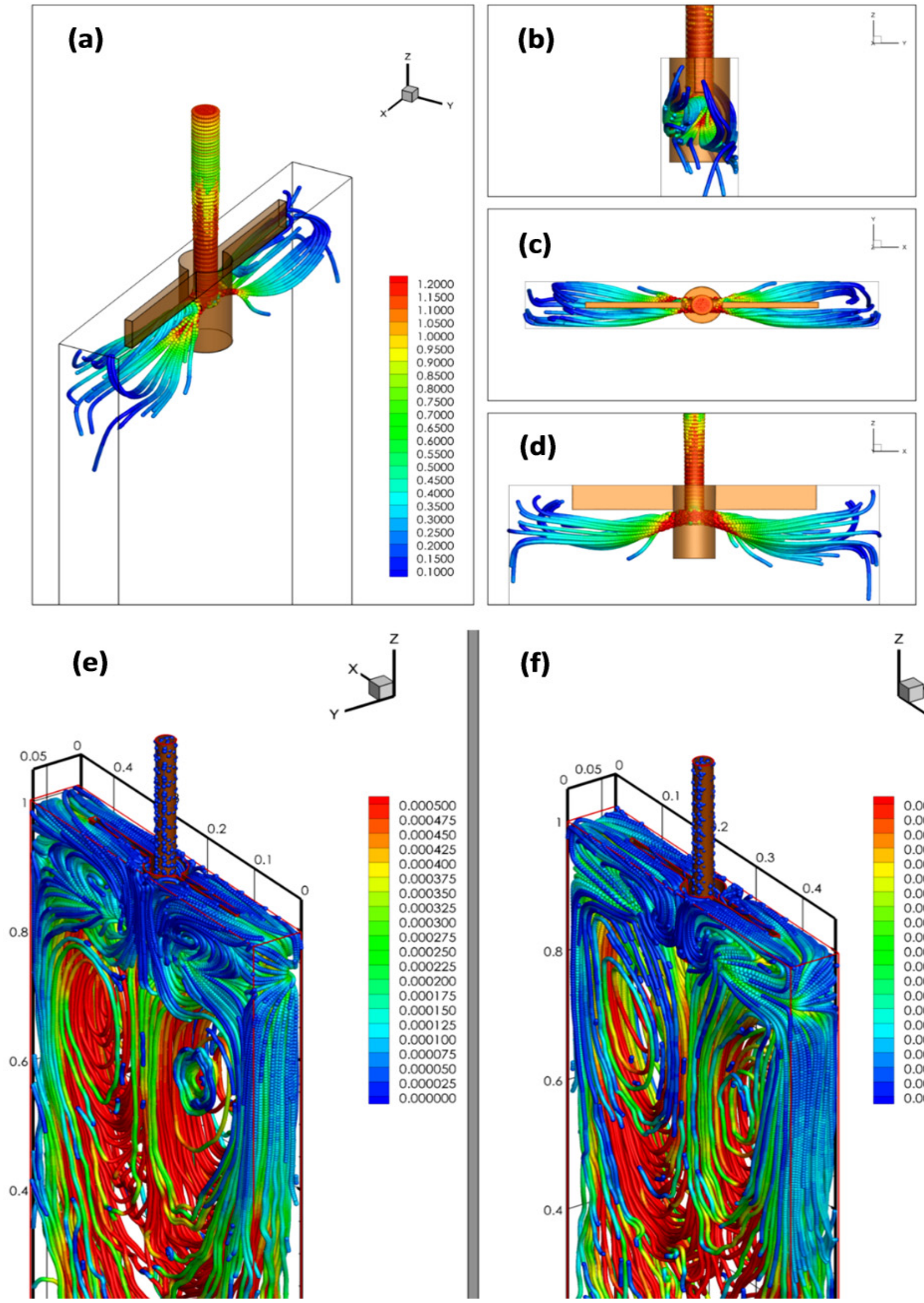

(f)<smiles></smiles>

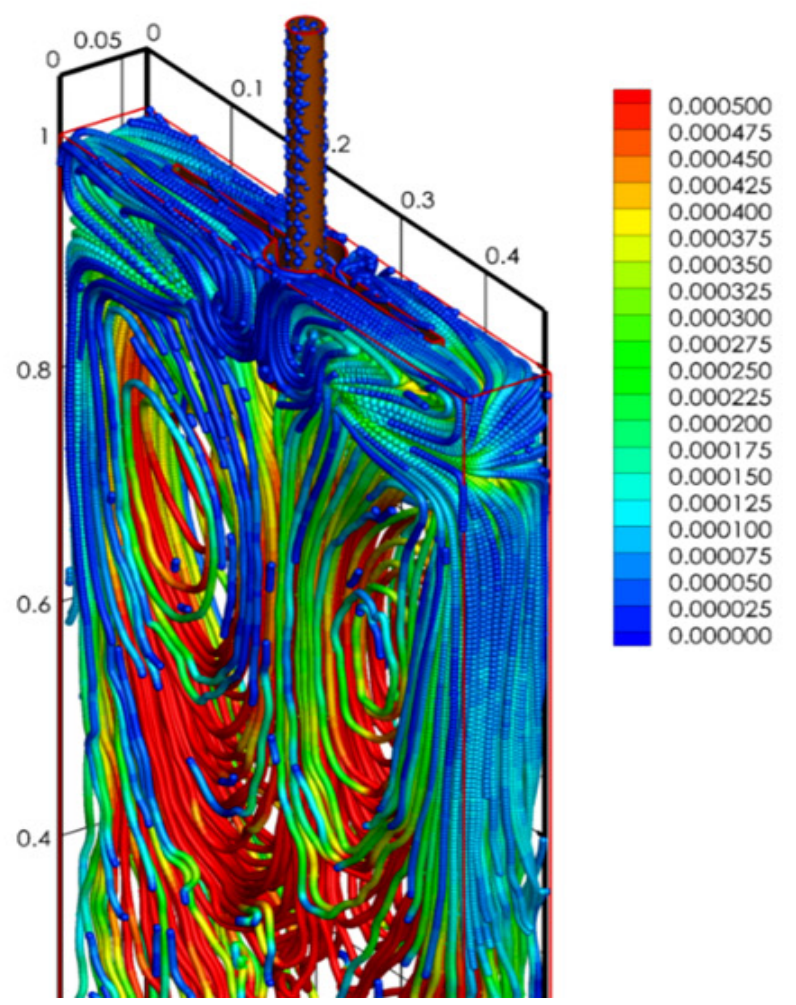

Fig. 7. Numerical simulation of flow pattern under transient condition for Model A, jets emerging from the outlets ports of the SEN, (a) isometric view, (b) lateral view, (c) upper view, (d) front view, units are $\left[\mathrm{m} \cdot \mathrm{s}^{-1}\right]$. Fluid flow pattern inside the mold, (e) isometric view front-wall oriented of mold (f) isometric view back wall oriented of mold, units are $\left[\mathrm{m}^{2} \cdot \mathrm{s}^{-1}\right]$ 

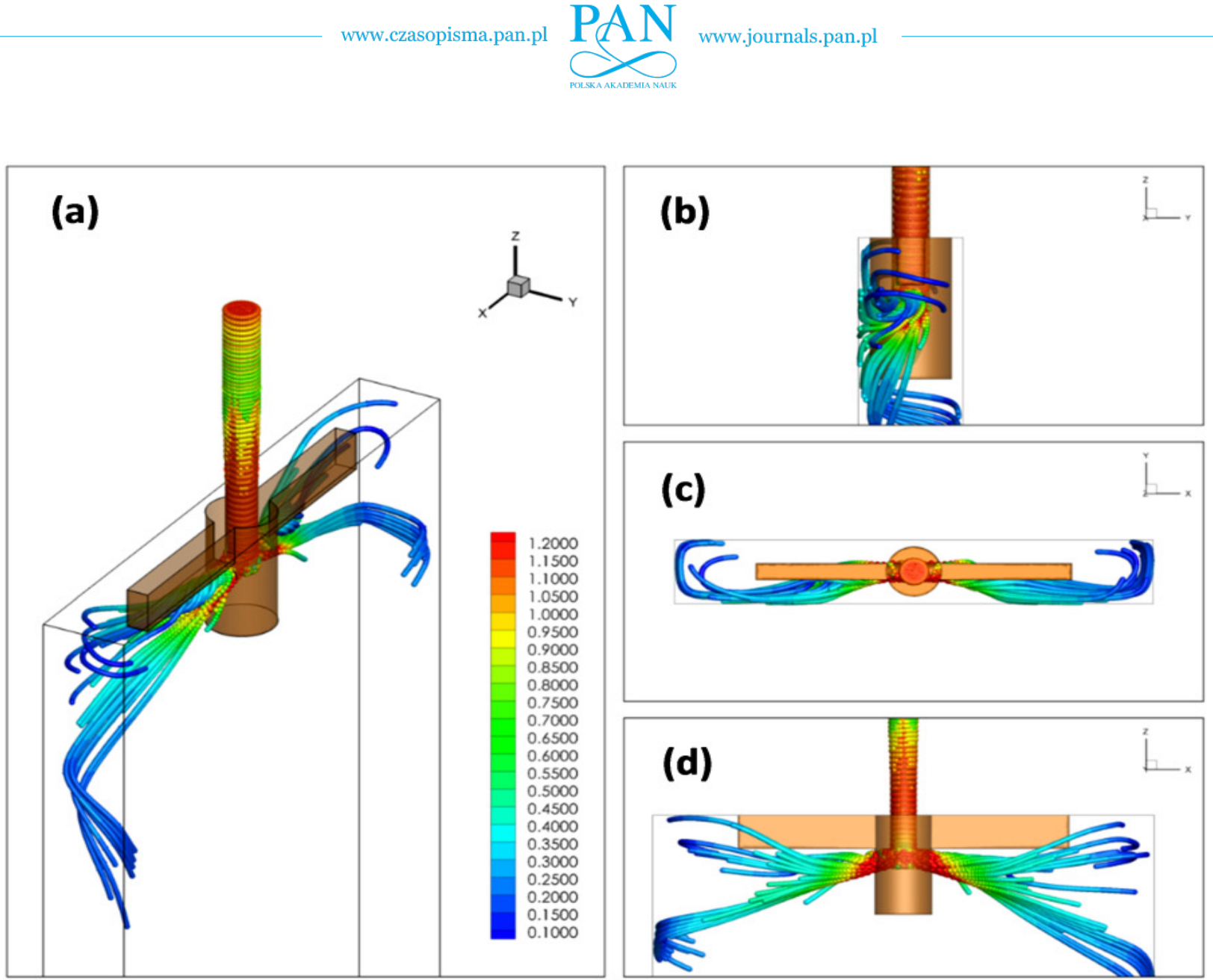

(e)

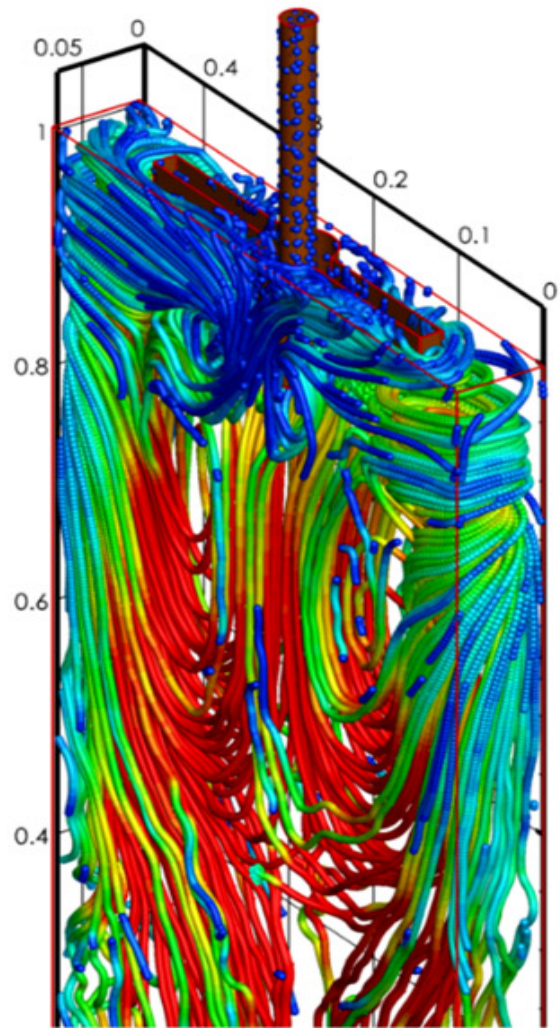

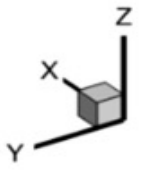

(f)<smiles></smiles>

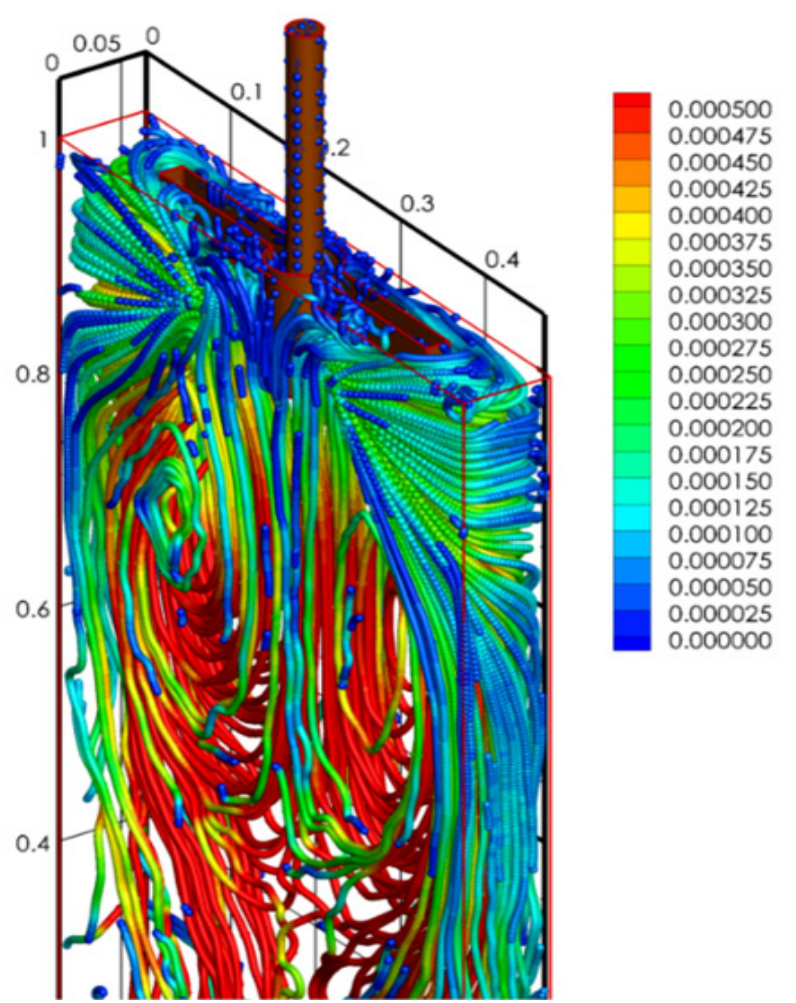

Fig. 8. Numerical simulation of flow pattern under transient condition for Model B, jets emerging from the outlets ports of the SEN, (a) isometric view, (b) lateral view, (c) upper view, (d) front view, units are $\left[\mathrm{m} \cdot \mathrm{s}^{-1}\right]$. Fluid flow pattern inside the mold, (e) isometric view front-wall oriented of mold (f) isometric view back wall oriented of mold, units are $\left[\mathrm{m}^{2} \cdot \mathrm{s}^{-1}\right]$ 

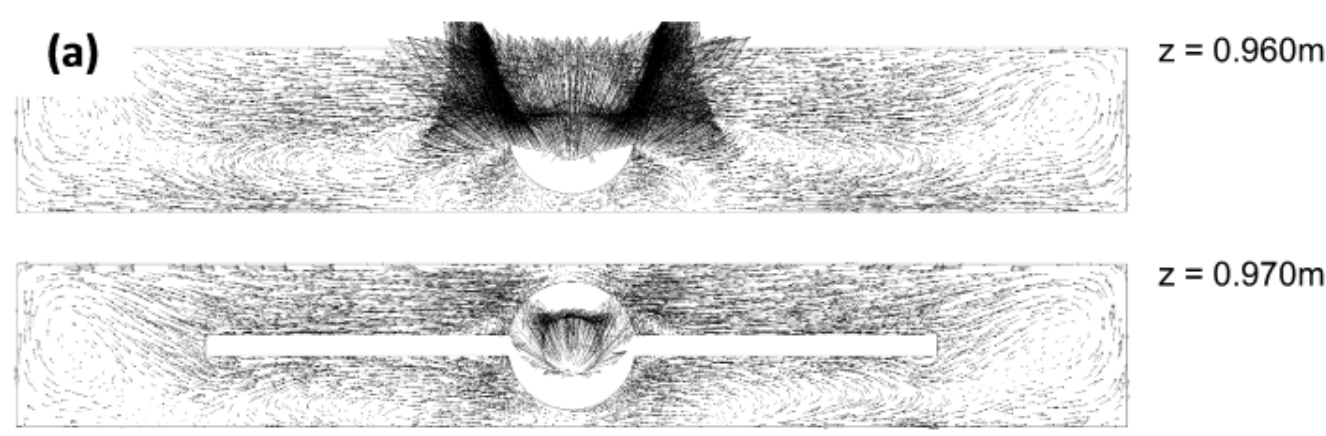

$z=0.970 m$
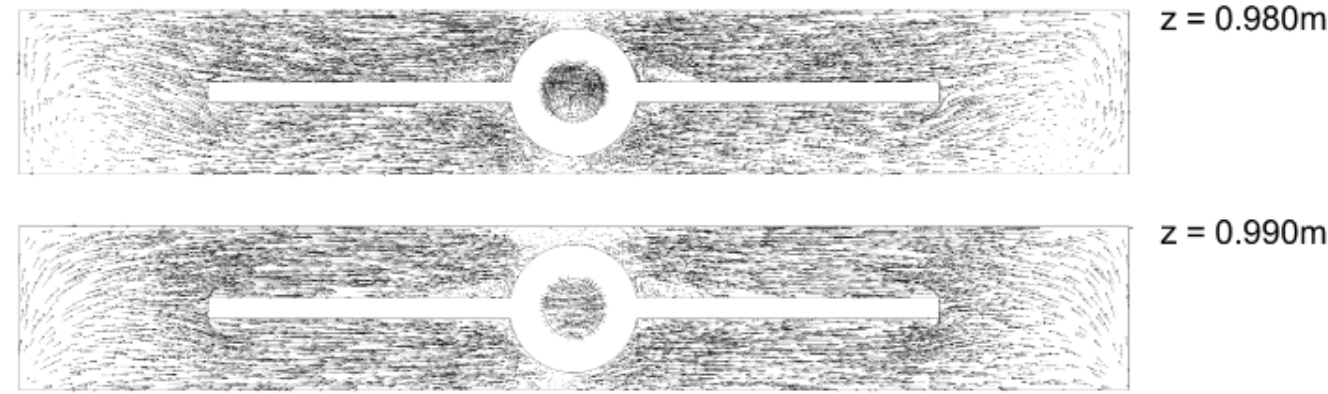

$z=0.990 m$
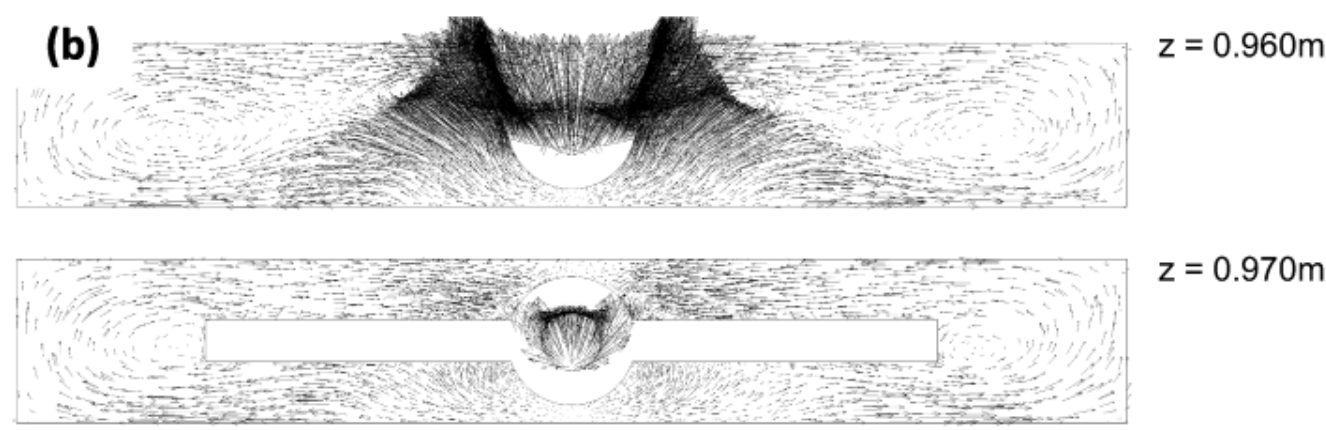

$z=0.970 m$
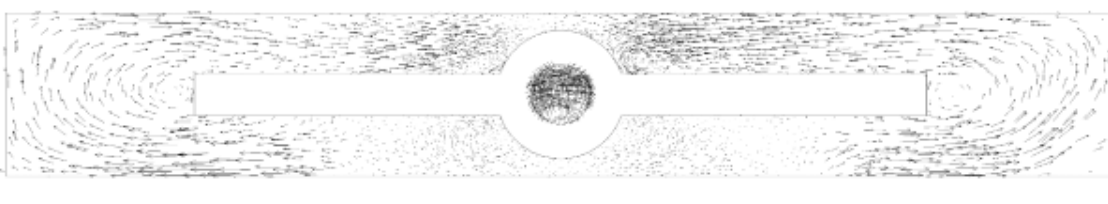

$z=0.980 m$

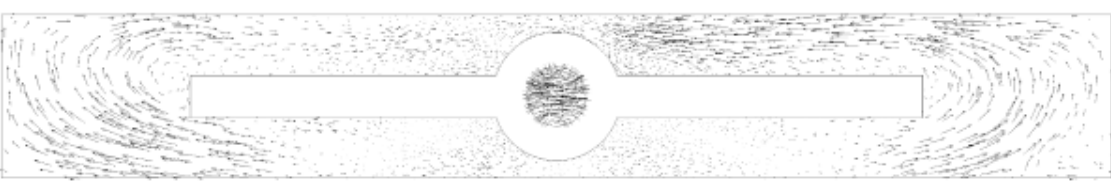

$z=0.990 m$

Fig. 9. Velocity vectors at four horizontal planes in the upper zone of the mold for (a) Model A nozzle and (b) Model B nozzle, under transient conditions. The length of the arrows is proportional to the fluid velocity magnitude at that point

Figure 10 shows that the recirculation zones located in the gaps between the extremities of the flow modifiers and the narrow walls of the mold have low intensity. This figure also confirms that Model C nozzle fulfills its original purpose. It is observed that for heights $\mathrm{Z}$ of $0.970 \mathrm{~m}, 0.980 \mathrm{~m}$ and $0.990 \mathrm{~m}$, almost all of the flow on both sides of the modifier is directed toward the nozzle.

Figures 10 shows the pattern of fluid flow in the mold has the four zones of recirculation pattern that characterizes the double-roll (DR) pattern. Furthermore, it is observed that the flow pattern produced by this nozzle is more symmetric with respect to the plane YZ than that obtained with the Model A nozzle.

\subsection{Model D nozzle under transient conditions}

As in the Model B nozzle, the increase of the thickness of modifier, pushes against one of the wide walls of the mold the jets emerging from the output ports of the nozzle, as is shown in Figure 11. Again, this behavior is totally unacceptable since it degrades the quality of the solidified layer.

Figure 12b shows that the performance of the Model D nozzle is better than that of the Model B nozzle, however, remains unacceptable. In addition, this figure shows that only very near the top of the mold ( $\mathrm{Z}$ height equal to $0.990 \mathrm{~m}$ ), almost all the 

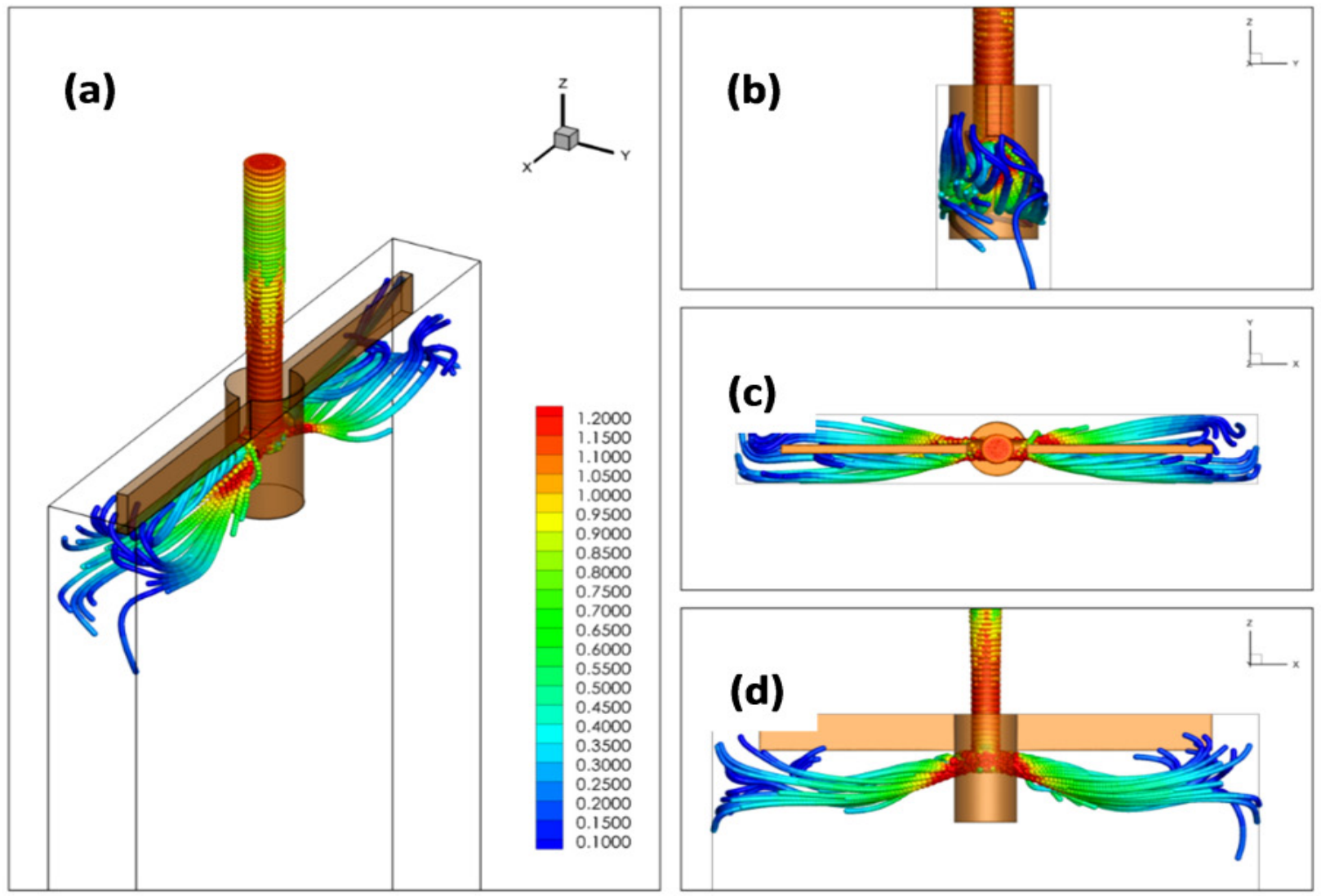

(c)
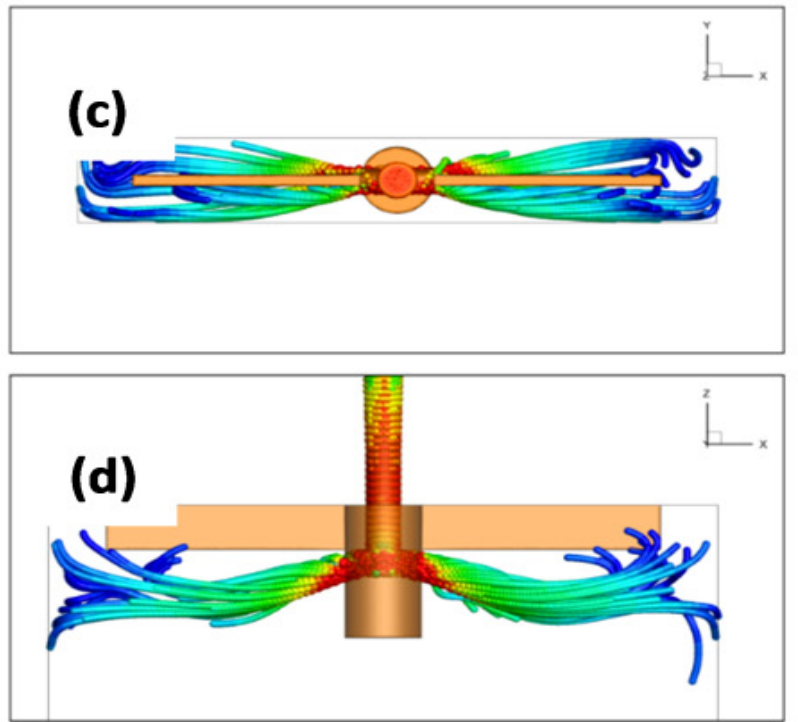

(e)

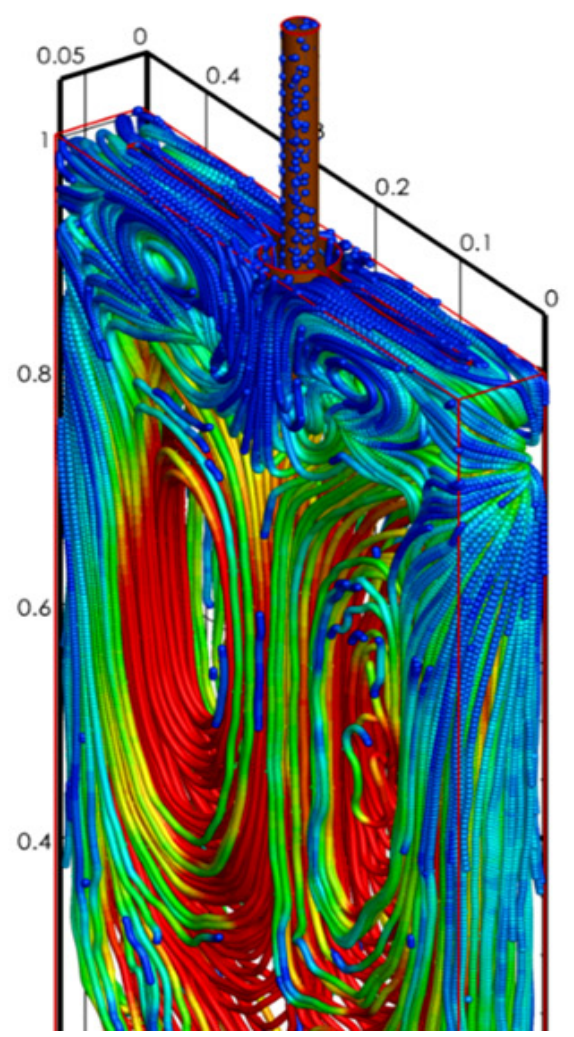

(f)<smiles></smiles>

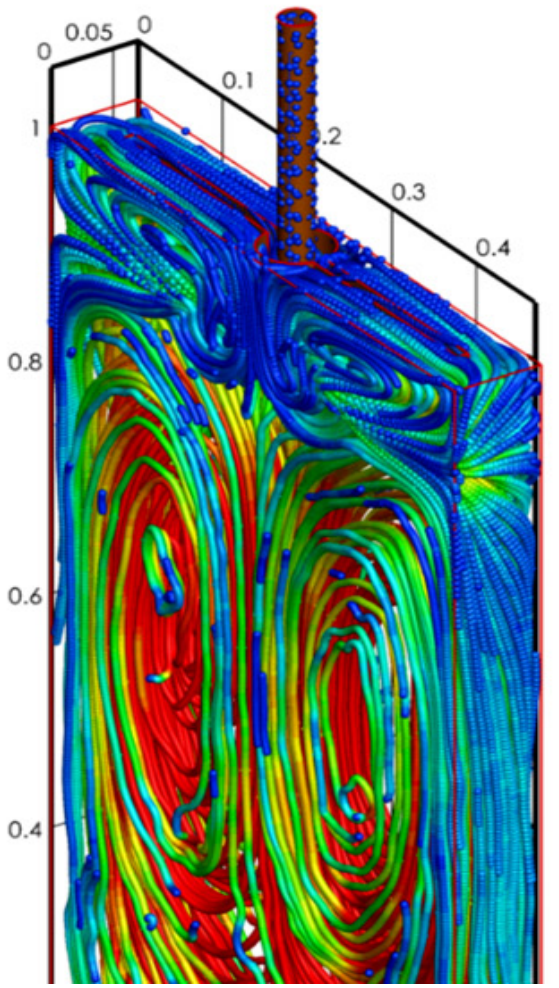

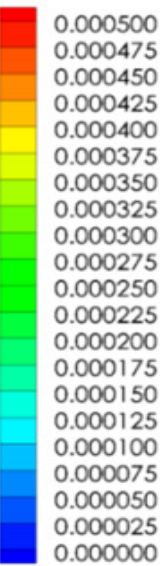

0.000500 .000475 0.000425 000400 000375 000325 0.000300 0.000225 0.000200 0.000150 0.000050 0.000000

Fig. 10. Numerical simulation of flow pattern under transient condition for Model C jets emerging from the outlets ports of the SEN, (a) isometric view, (b) lateral view, (c) upper view, (d) front view, units are $\left[\mathrm{m} \cdot \mathrm{s}^{-1}\right]$. Fluid flow pattern inside the mold, (e) isometric view front-wall oriented of mold (f) isometric view back wall oriented of mold, units are $\left[\mathrm{m}^{2} \cdot \mathrm{s}^{-1}\right]$ 

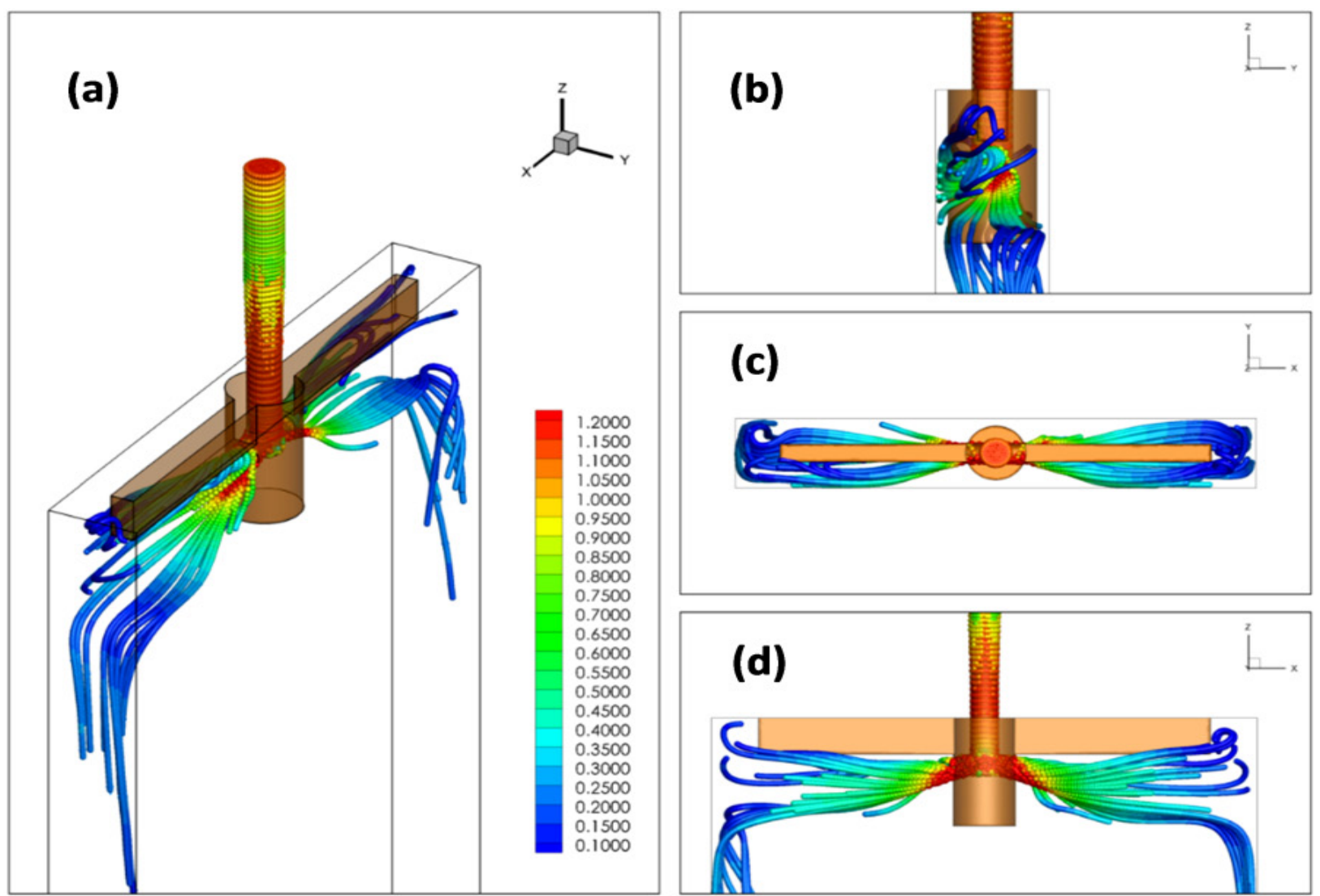

(e)

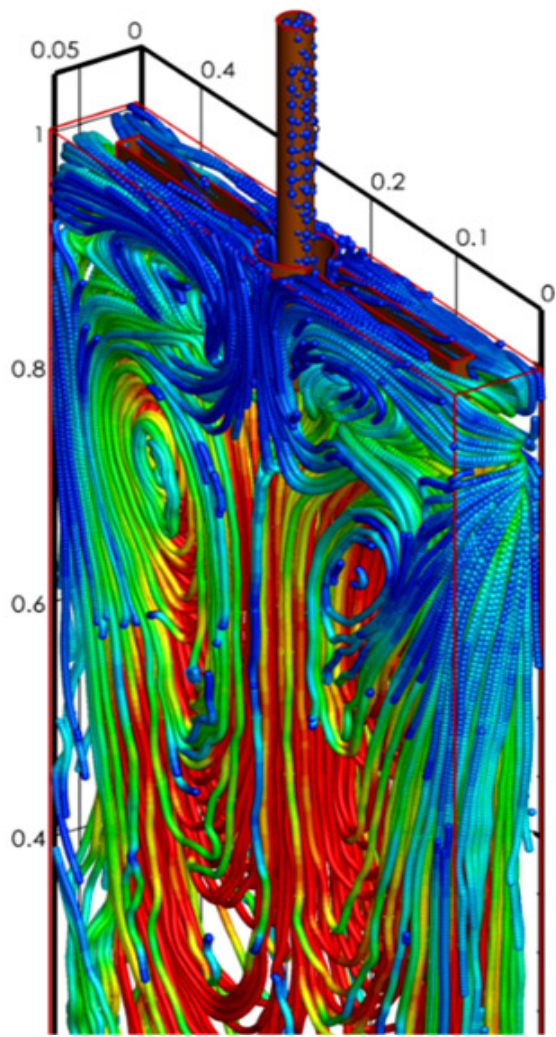

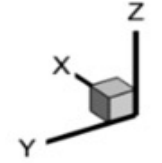

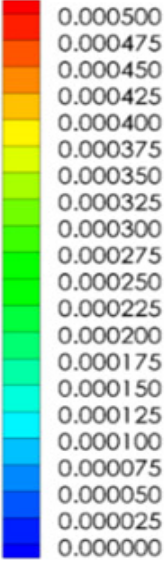

(f)<smiles></smiles>

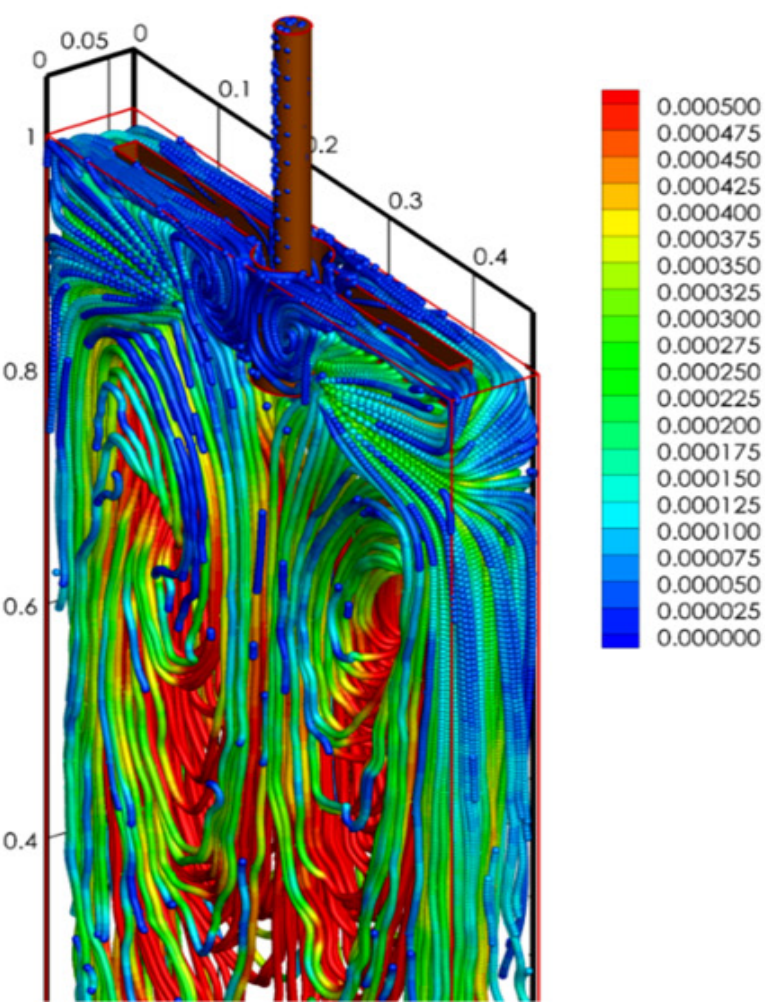

Fig. 11. Numerical simulation of flow pattern under transient condition for Model D jets emerging from the outlets ports of the SEN, (a) isometric view, (b) lateral view, (c) upper view, (d) front view, units are $\left[\mathrm{m} \cdot \mathrm{s}^{-1}\right]$. Fluid flow pattern inside the mold, (e) isometric view front-wall oriented of mold (f) isometric view back wall oriented of mold, units are $\left[\mathrm{m}^{2} \cdot \mathrm{s}^{-1}\right]$ 
fluid on both sides of the modifier directs toward the nozzle. Below this height, a large part of the flow on both sides of the modifier has an upward direction.

Figure 11 shows that this nozzle generates inside the mold the four recirculation zones that characterize the double-roll (DR) pattern. However, Figures 11 and 12b demonstrate that the behavior in each mold halves is very different in respect the XZ plane. Based on these results, we can assume that this nozzle will generate more fluctuations in the level of liquid steel in the mold in comparison to that generated by Model C nozzle.

\subsection{Model E nozzle under transient conditions}

This configuration cannot be implemented in industry because it will degrade the formation of the solidified shield of steel in meniscus. However, this model allows to analyze the influence of the gap between the edge of the modifier and the narrow wall of the mold.

Figure 13 shows that the jets emerging from the outlet ports of the nozzle are pushed against one of the wide walls of the mold. However, it is also noted that the jet does not scatter as much as in Models B and D nozzles. Figure 15a shows that the behavior in each mold halves is very different in respect the $\mathrm{XZ}$ plane.

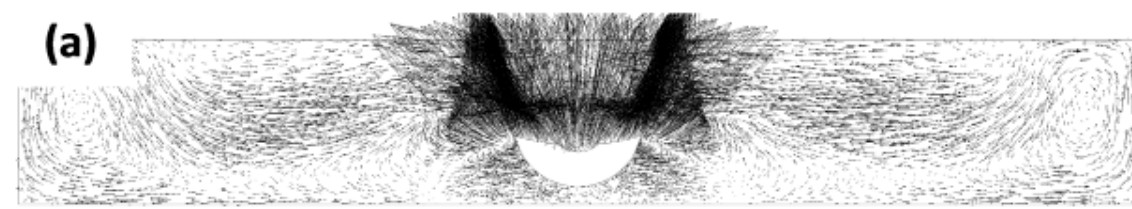

$z=0.960 m$

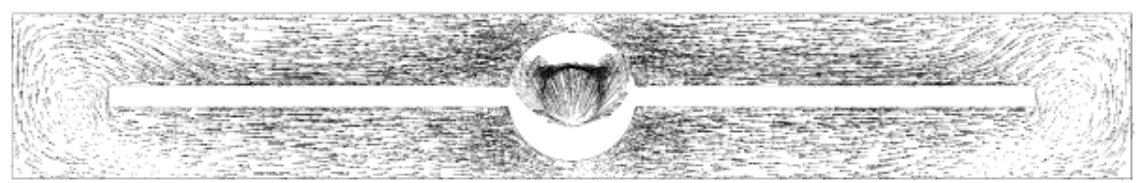

$z=0.970 m$

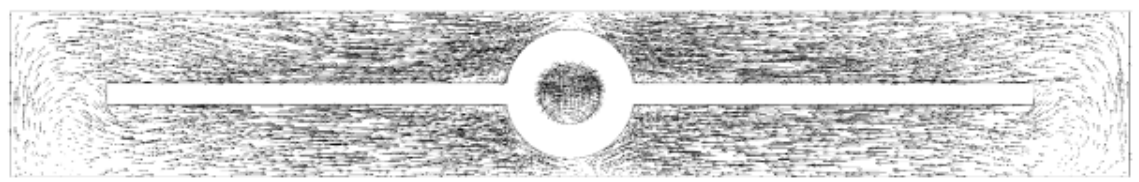

$z=0.980 m$

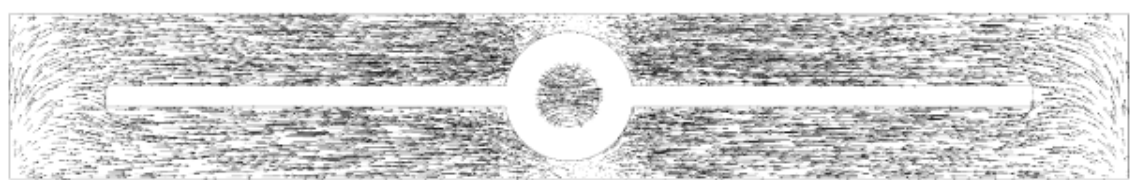

$z=0.990 m$

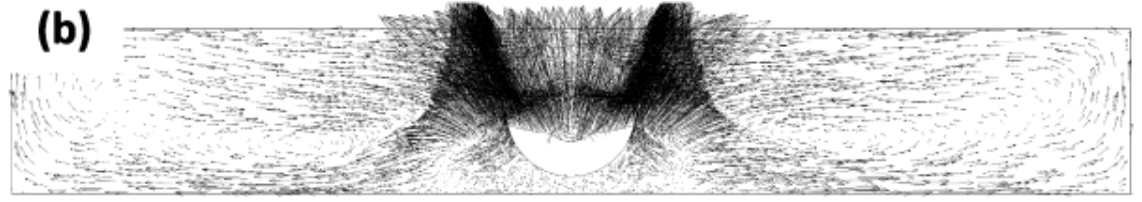

$z=0.960 m$

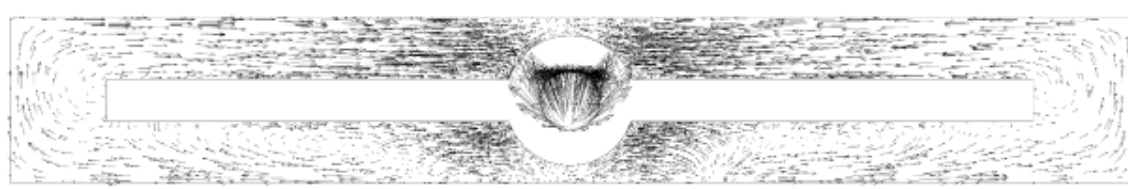

$z=0.970 m$
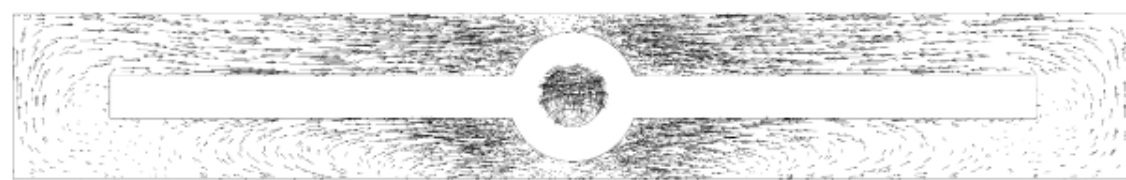

$z=0.980 m$
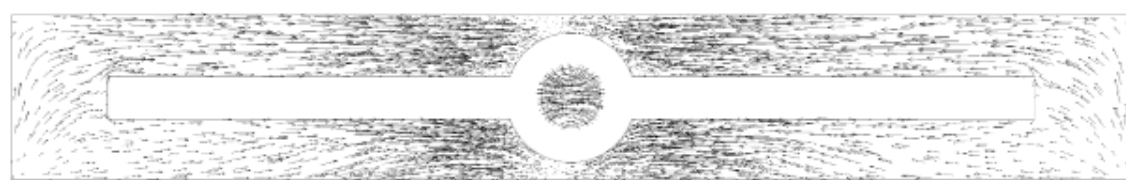

$z=0.990 m$

Fig. 12. Velocity vectors at four horizontal planes in the upper zone of the mold for (a) Model C nozzle and (b) Model D nozzle, under transient conditions. The length of the arrows is proportional to the fluid velocity magnitude at that point 

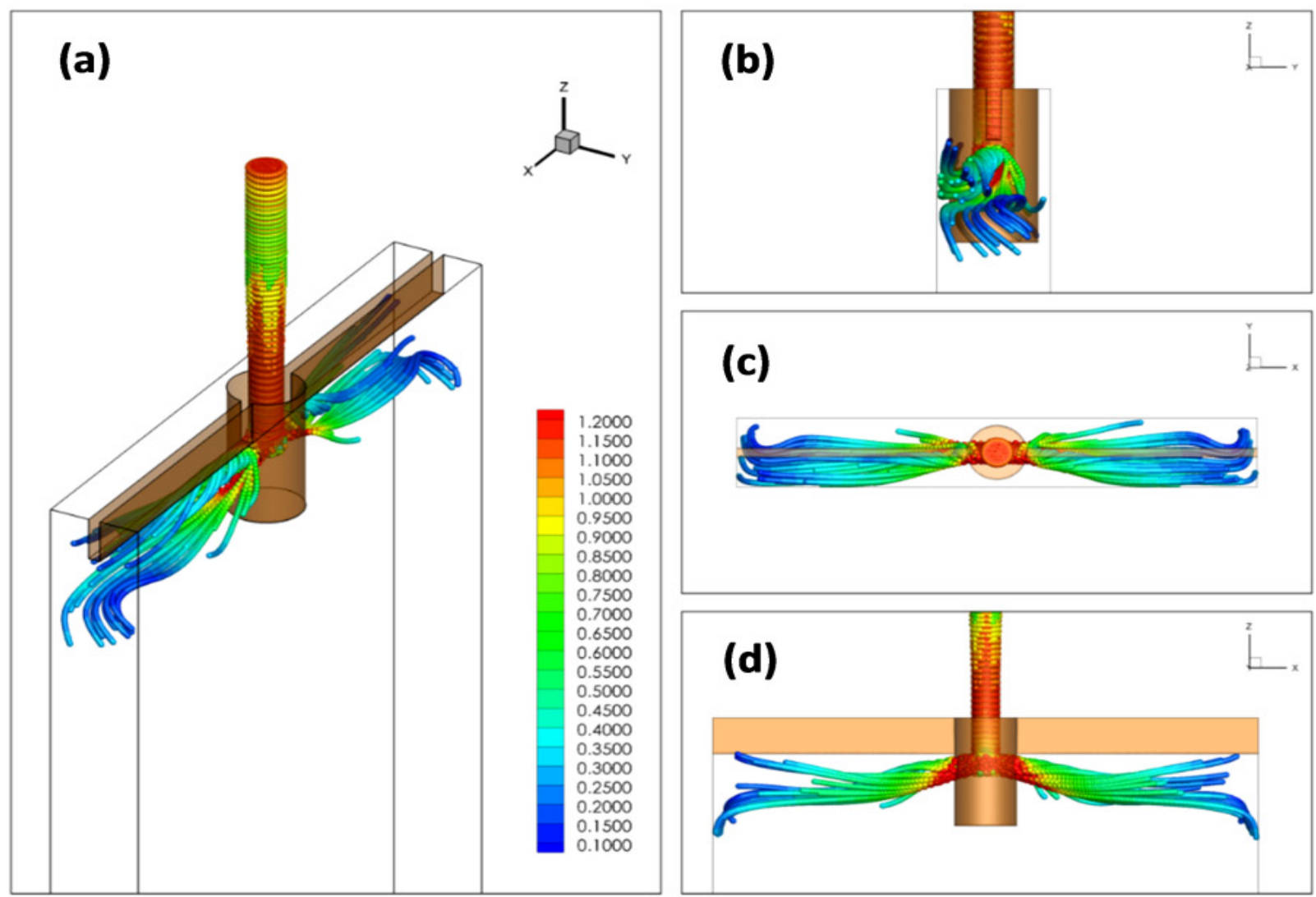

(e)

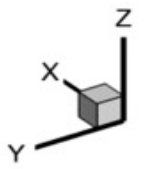

(f)
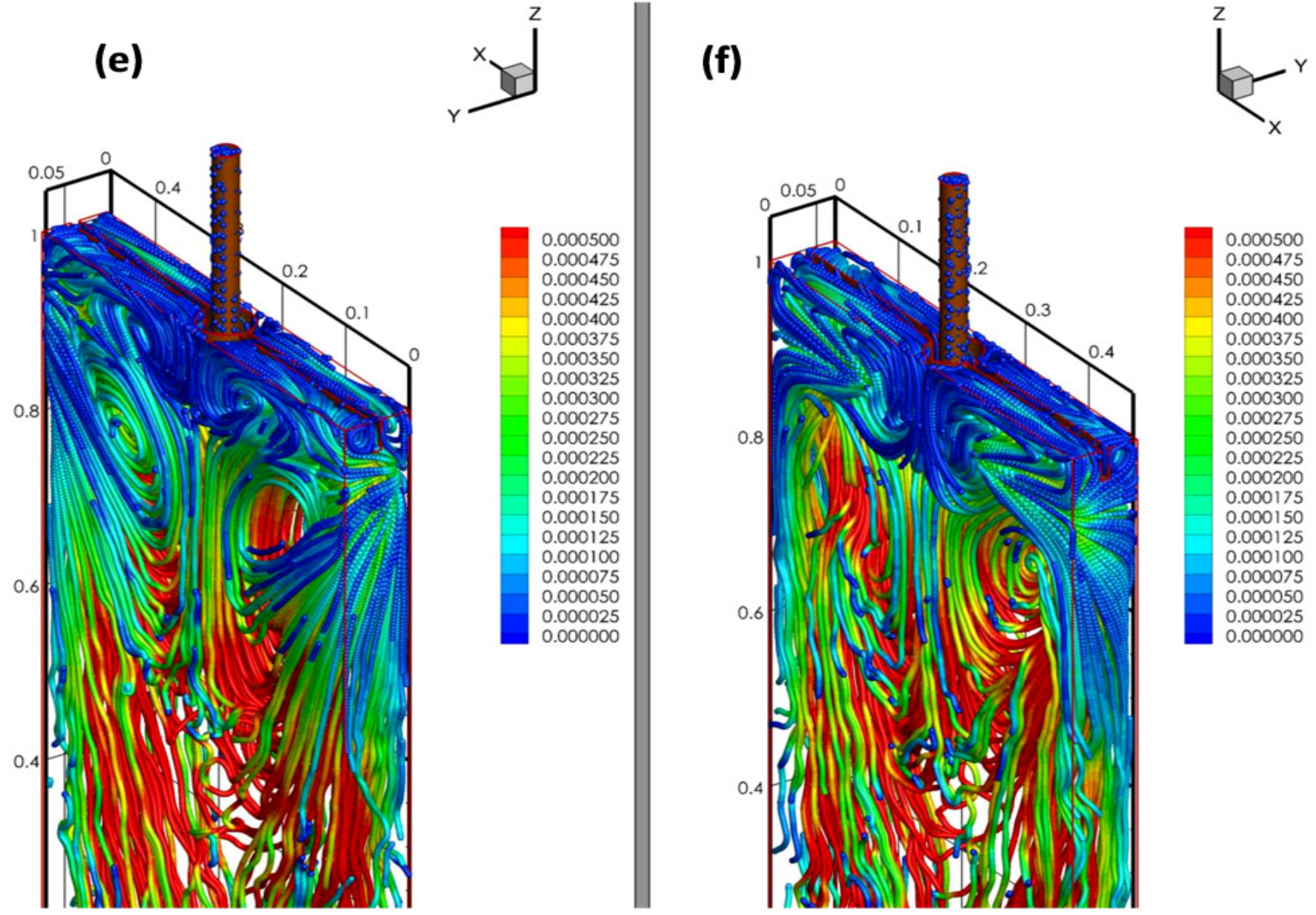

0.000000

Fig. 13. Numerical simulation of flow pattern under transient condition for Model E jets emerging from the outlets ports of the SEN, (a) isometric view, (b) lateral view, (c) upper view, (d) front view, units are $\left[\mathrm{m} \cdot \mathrm{s}^{-1}\right]$. Fluid flow pattern inside the mold, (e) isometric view front-wall oriented of mold (f) isometric view back wall oriented of mold, units are $\left[\mathrm{m}^{2} \cdot \mathrm{s}^{-1}\right]$ 

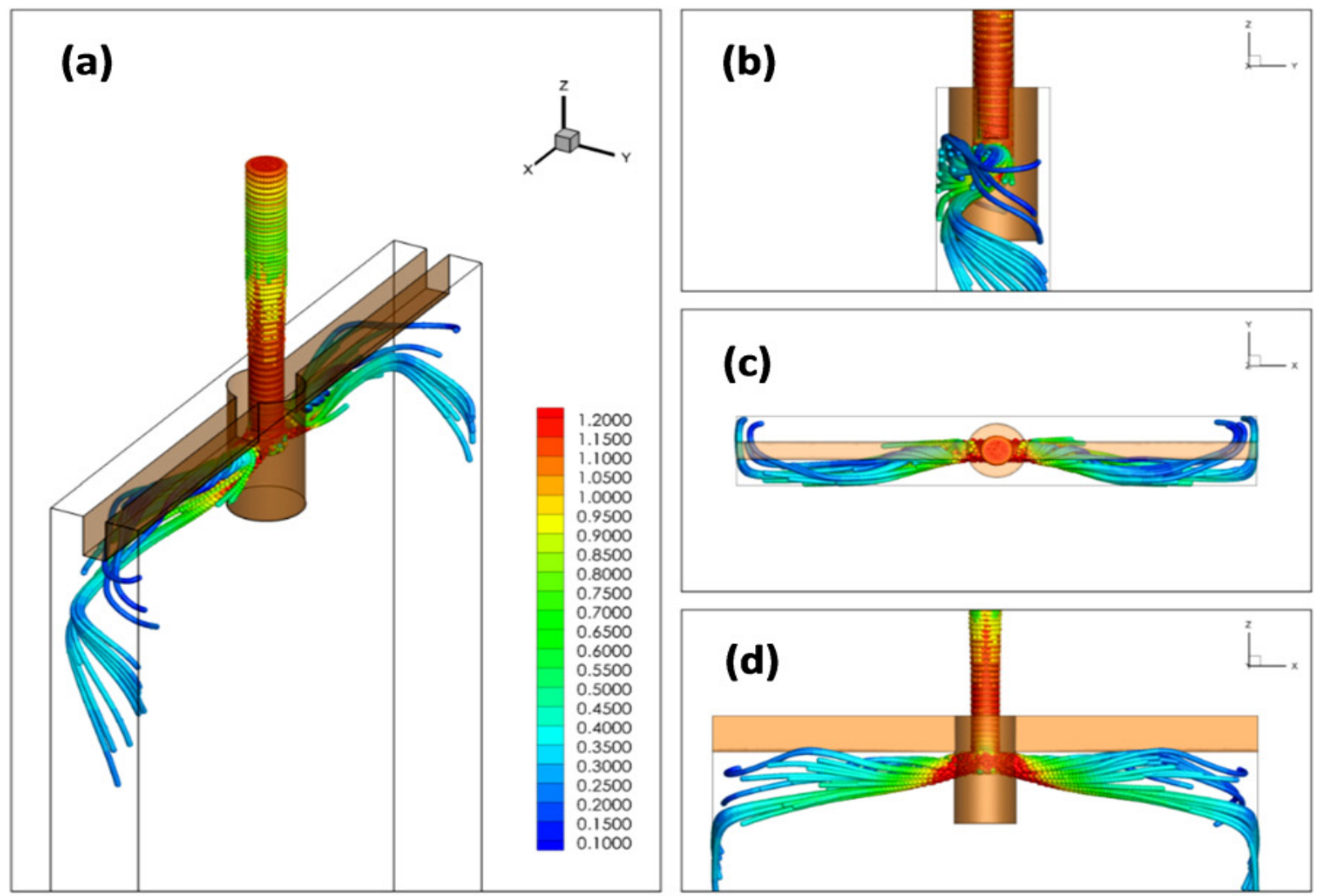

(e)

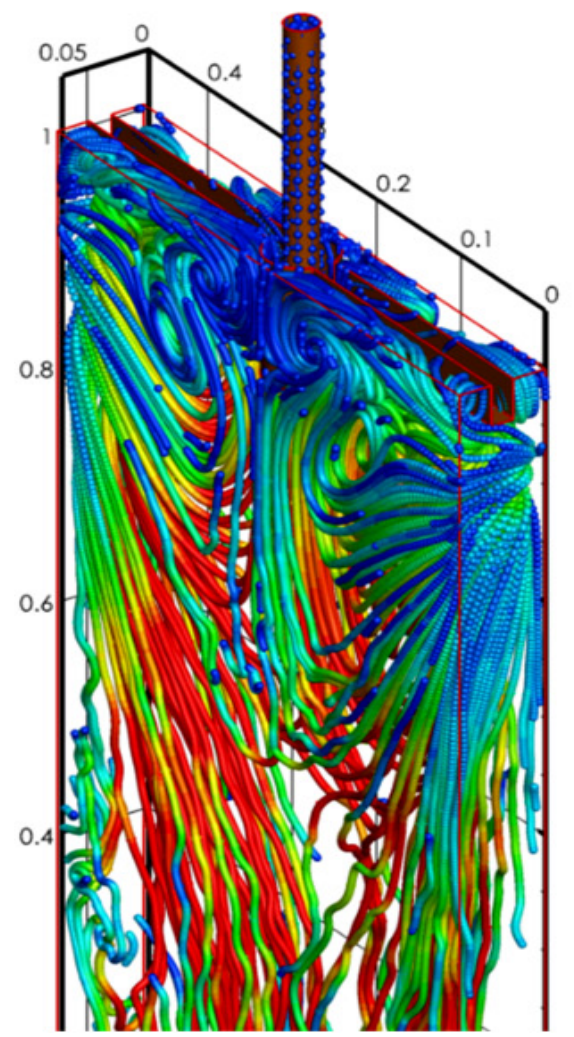

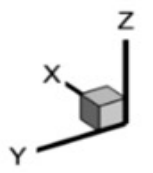

(f)<smiles></smiles>

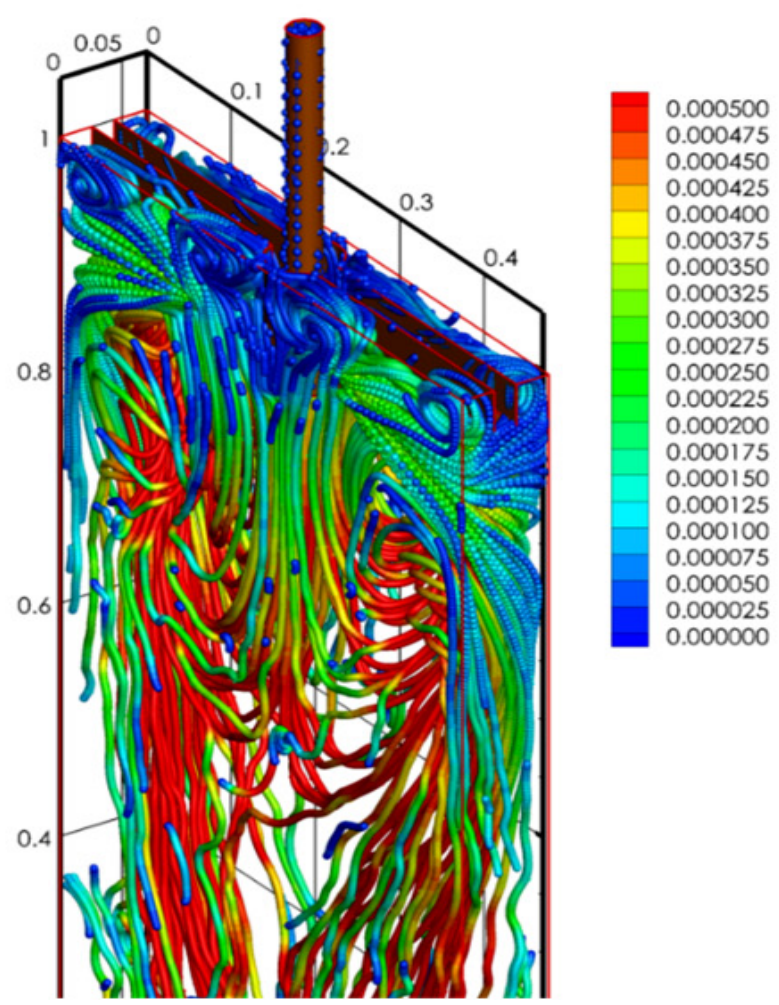

Fig. 14. Numerical simulation of flow pattern under transient condition for Model F jets emerging from the outlets ports of the SEN, (a) isometric view, (b) lateral view, (c) upper view, (d) front view, units are $\left[\mathrm{m} \cdot \mathrm{s}^{-1}\right.$. Fluid flow pattern inside the mold, (e) isometric view front-wall oriented of mold (f) isometric view back wall oriented of mold, units are $\left[\mathrm{m}^{2} \cdot \mathrm{s}^{-1}\right]$ 
These results suggest that the overall performance of the modifiers is inadequate. Almost all the fluid directs toward the nozzle only on one of the halves and only near the top of the mold. There are vortices whose axis of rotation is perpendicular to the main axis of the nozzle at only one of the mold halves and close to the top.

In addition, Figure 13 shows a high degeneracy of the vortices or recirculation zones in the lower section of the mold. It is imperative to have these recirculation zones because they help to improve product quality by sending to the top of the mold those impurities that reach this area of the mold.

\subsection{Model F nozzle under transient conditions}

The nozzles for Models $\mathrm{E}$ and $\mathrm{F}$ have the same length, but the thickness of the modifiers for Model F nozzle is twice the thickness of the modifiers for Model F. Figure 14 confirms the trend observed for Models B and D nozzles, the increase of the thickness of the modifier, pushes against one of the wide walls of the mold the jets emerging from the output ports of the nozzle.

Figure $15 \mathrm{~b}$ also confirms that the performance of the nozzle Model E is atrocious and does not meet the original purpose of
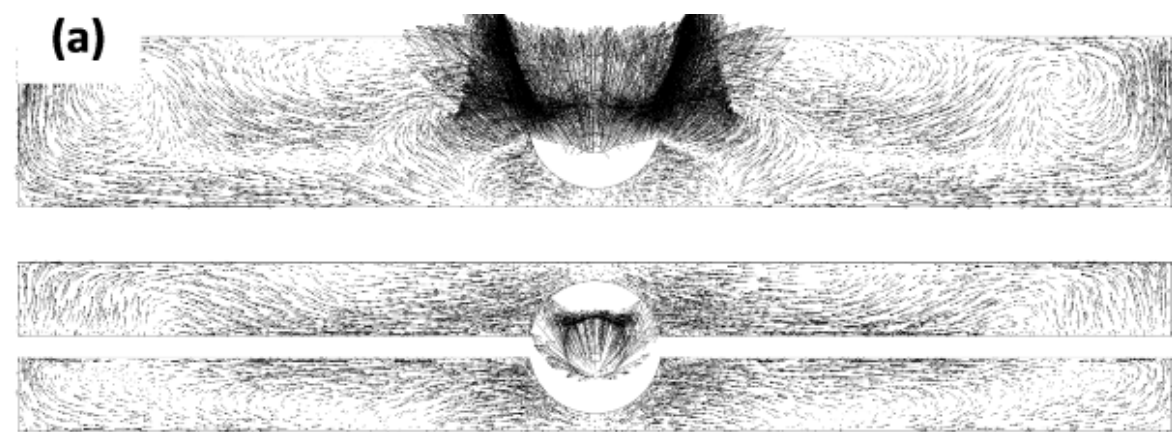

$z=0.970 m$

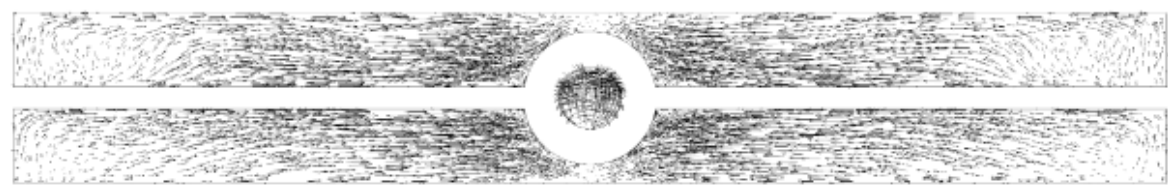

$z=0.980 m$

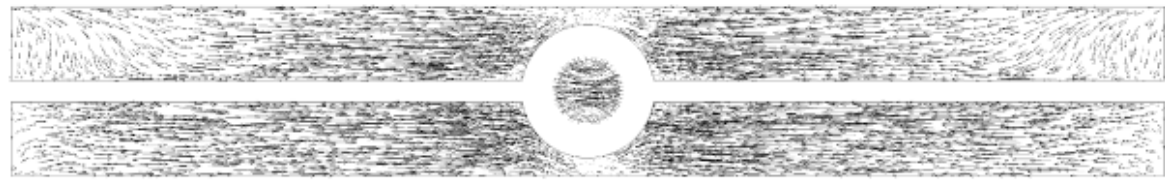

$z=0.990 m$

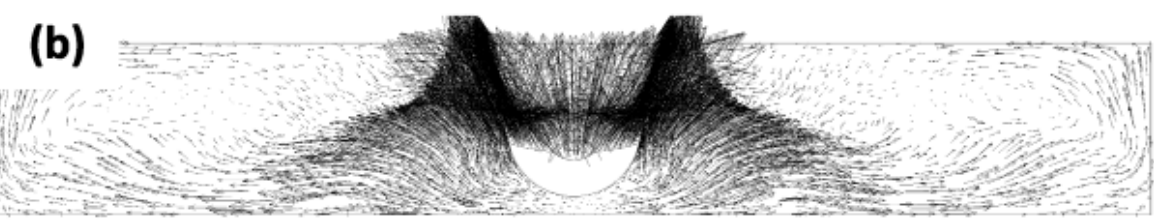

$z=0.960 m$

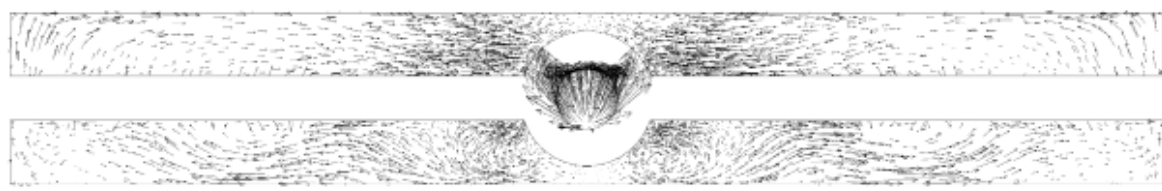

$z=0.970 m$

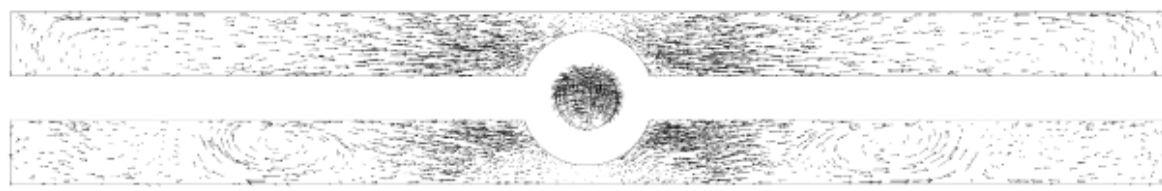

$z=0.980 m$

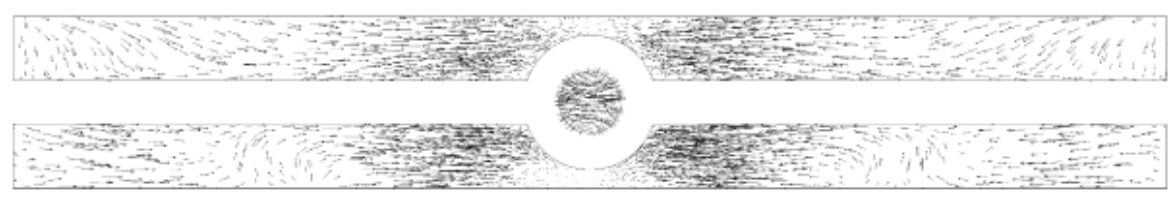

$z=0.990 m$

Fig. 15. Velocity vectors at four horizontal planes in the upper zone of the mold for (a) Model E nozzle and (b) Model F nozzle, under transient conditions. The length of the arrows is proportional to the fluid velocity magnitude at that point 
flow modifiers. In the upper section of the mold, the behavior in each of the mold halves is very different in respect the $\mathrm{XZ}$ plane. This figure reveals many recirculation zones in this section of the mold. For this reason, we can assume that this nozzle will generate a considerable amount of fluctuations in the level of liquid steel in both halves of the mold.

\section{Physical evaluation of the fluid flow modifiers}

The liquid level measurements were taken using an E-tape Liquid Level Sensor from Milone Technologies. The level sensor has a resistive value that changes with the level of the water. The resistive value of the sensor is inversely proportional to the height of the water; that is, the lower the liquid level is, the higher the resistance value. The main feature of this sensor is that does not employ any mechanical flotation device and easily interfaces with electronic circuit to obtain a voltage value proportional to the water level and with that develops the measurement and controls the scaled model. For this work, the sensor resolution and accuracy are sufficient.

However, these measurements will be compared with PIV measurements in future works. PIV equipment obtain very high resolution measurements and could be used as a direct comparison with numerical simulation in order to obtain the validation.

Some of the results obtained in these physical experiments show that the performance of the sensor was sufficient for the scope of this study, because of the differences in liquid level is proportional to the measurements rate.

The physical experiment setup is shown in Figure 16, a rigid acrylic-made support is installed, on every liquid level sensor, also is possible to observe the mounting system of each of the liquid sensors over the front and back walls of the mold. The mounting is installed on the upper edge of the acrylic-made prism (mold) over a sliding device to obtain measurements along the edge of the mold.

In this work was considered the obtained measurements of liquid level sensor 1 and its position was near the narrow wall of the mold, because in this zone, the higher water level is observed.

Although the liquid level sensor is thin, it is an intrusive device and, therefore could modify the fluid flow pattern inside the mold. In addition, it was necessary to mount the level sensor on a support that avoid flexion of the sensor due to the flow streams generated inside the mold. For this reason, the liquid level was measured at a single position. In all cases, the sensor was placed $0.170 \mathrm{~m}$ of distance (the outer diameter of the nozzle) from the nozzle, and the sampling rate was 10 samples per second.

The results of the physical simulations are reported in Figure 17. The vertical axis indicates the values of the voltage signal generated by the liquid level sensor and its units are [mV]. These values are directly proportional to the height of the liquid level in the mold.

These results confirm almost all the findings raised through the numerical simulations discussed in the previous section.

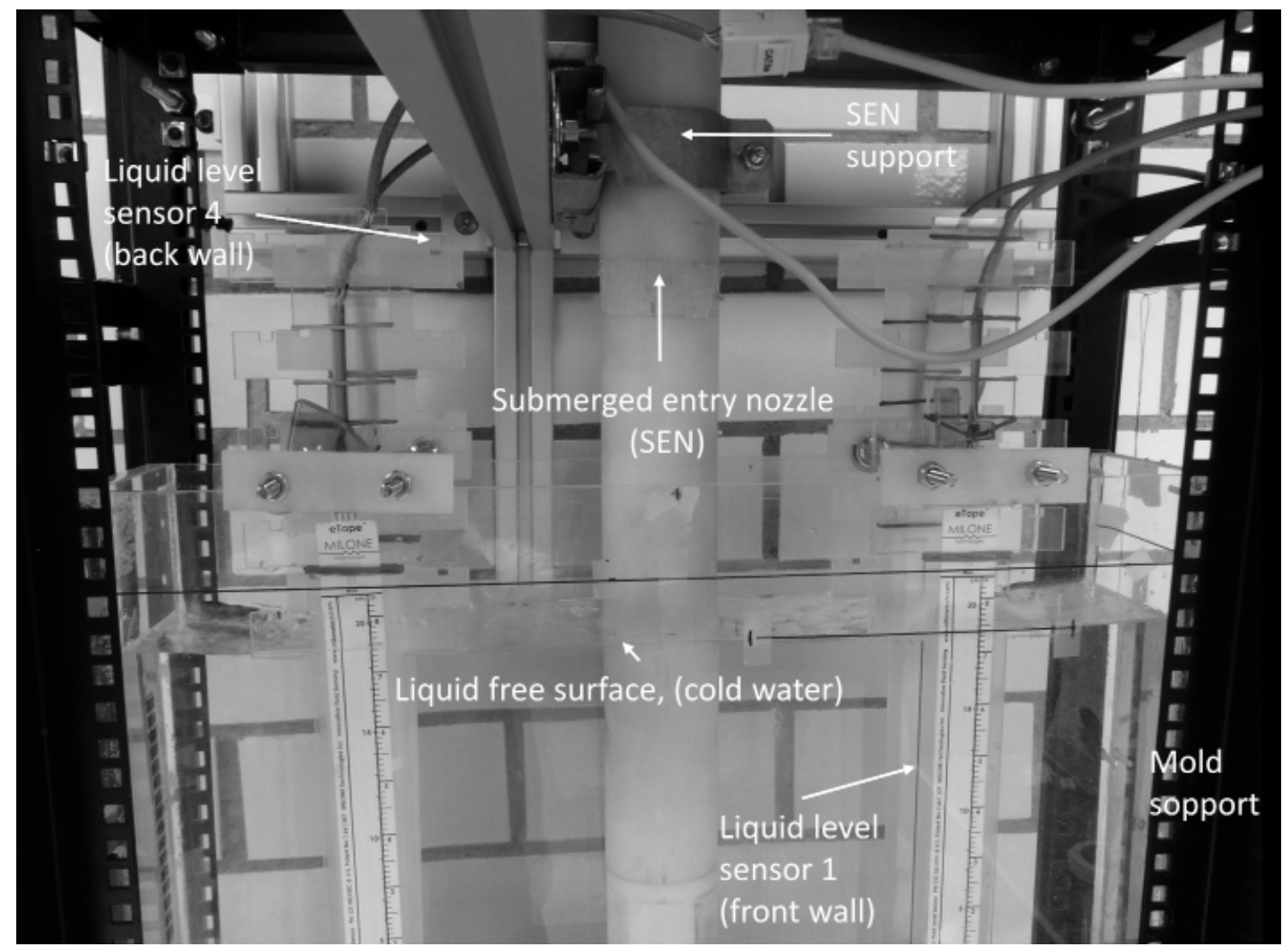

Fig. 16. Physical experiment setup. 1:1/3 Scaled model, traditional bifurcated submerged entry nozzle (SEN) and mold instrumented with liquid level sensors 
(a)

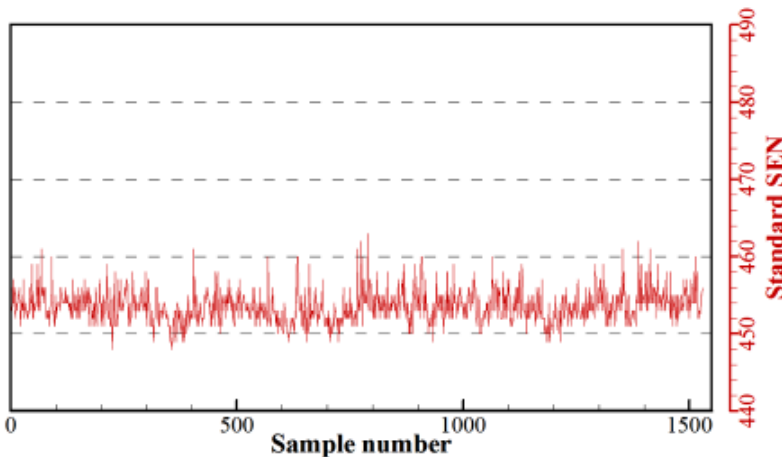

(b)

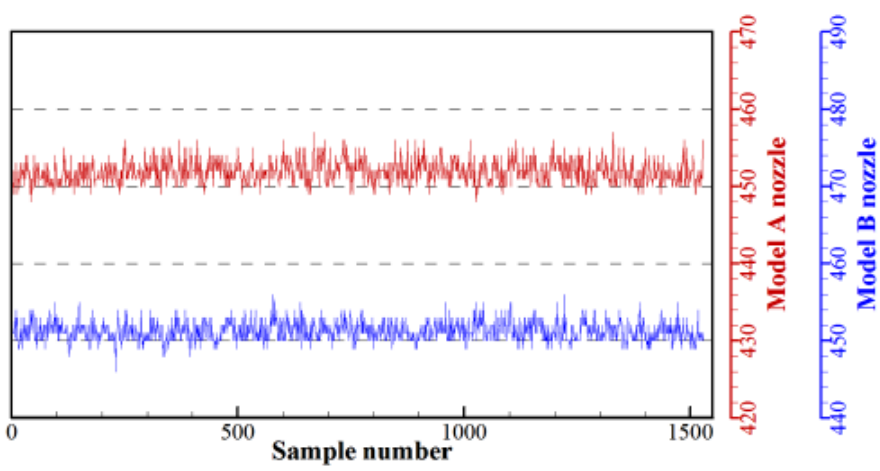

(d)
Model E nozzle Model F nozzle (c)

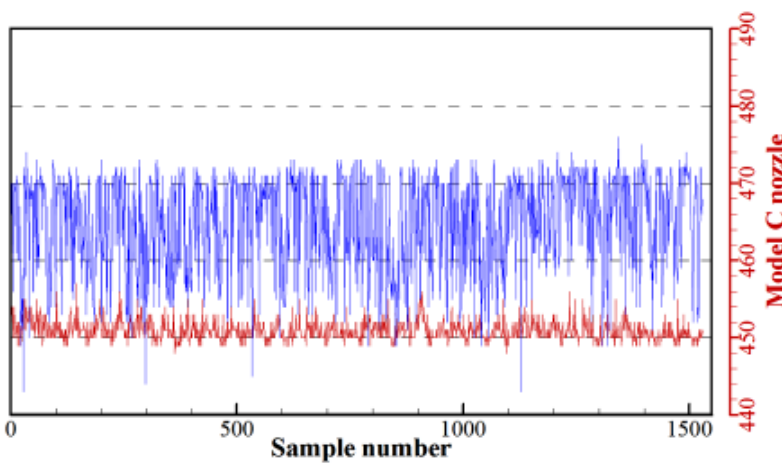

Model C nozzle Model D nozzle
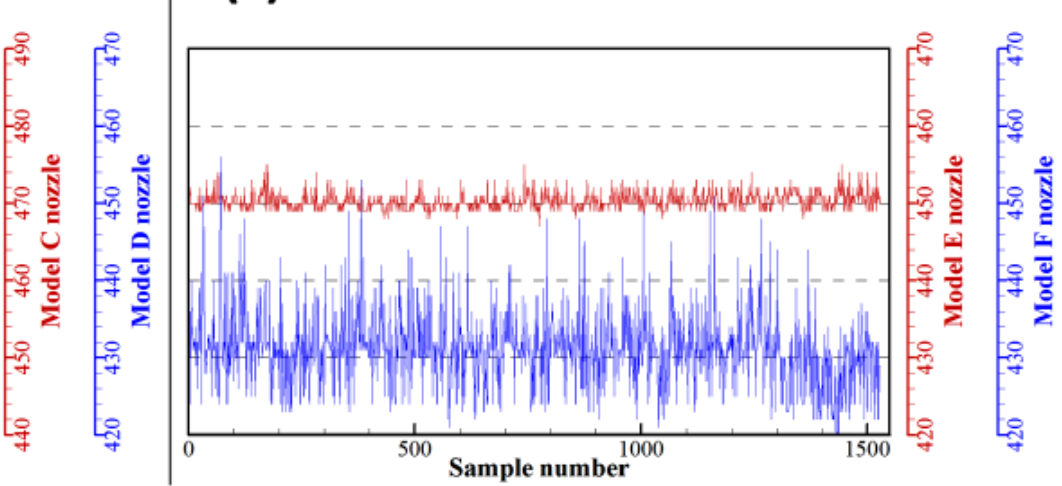

Fig. 17. Liquid level measurements for (a) standard Model, (b) A and B Models, (c) C and D Models, (d) E and F Models. The units of the vertical axis are $[\mathrm{mV}]$

Figures 17c and 17d show that Model D and F nozzles have the worst performance. Figures 17b, 17c and 17d show that Models $\mathrm{A}, \mathrm{B}, \mathrm{C}$ and $\mathrm{E}$ nozzles indeed attenuate the fluctuations of the liquid level compared with the standard SEN, whose performance is shown Figure 17a.

Figure 17b suggests that Model B nozzle has a better performance than Model A nozzle. A result, that this figure does not show, is that the turbulence in the central zone of the mold is much more intense for the Model B nozzle, compared to that observed for the Model A nozzle.

Figure $17 \mathrm{~d}$ show that smaller fluctuations in the liquid level were recorded when the Model E nozzle was employed, this result is confirmed in Figure 18 where is observed that the standard distribution of Model E nozzle is the closest to standard nozzle at steady condition.

\section{Conclusions}

The numerical and physical simulations presented in this study corroborated that the performance a standard nozzle can be improved by attaching fluid flow modifiers at the external wall of the bifurcated nozzle. This work examined fluid flow modifiers of rectangular shape. The effect of the change in the thickness and the length of the modifier was analyzed. All the modifiers had the same height.

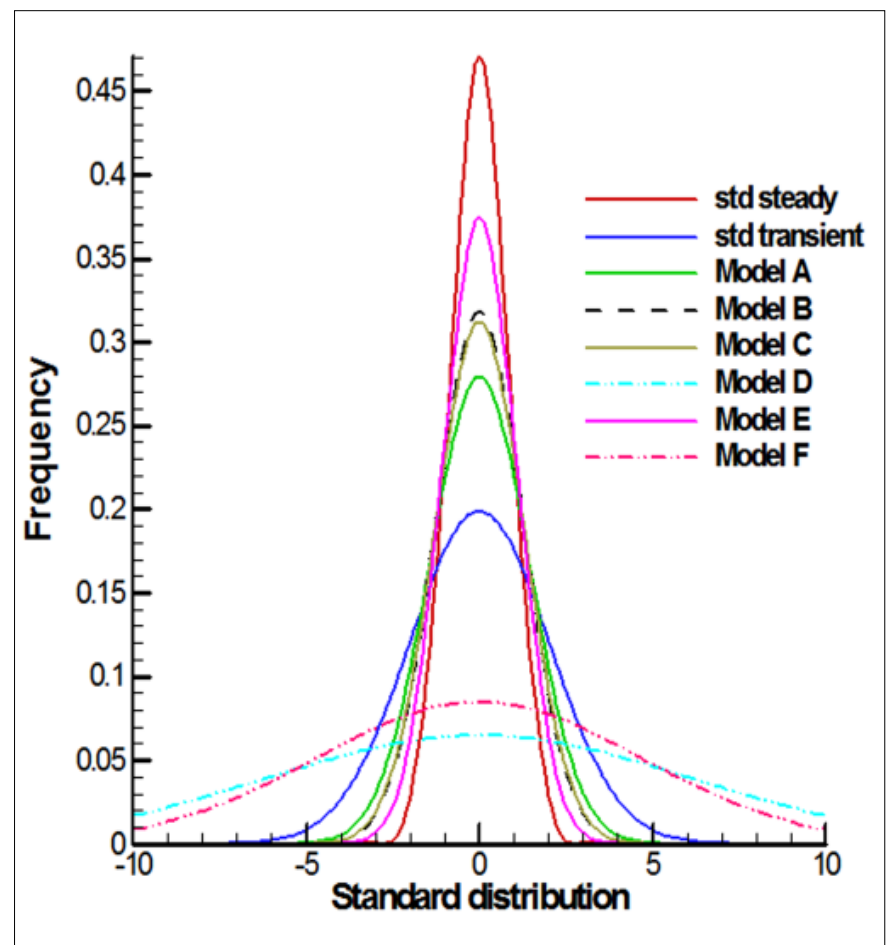

Fig. 18. Comparison of the standard distribution for liquid level measurements in physical experiment setup for standard, Model A, Model B, Model C, Model E and Model F nozzles 
Physical and numerical simulations showed that the physical dimensions of the flow modifiers significantly change the fluid flow pattern generated in the mold of continuous casting of steel.

The results of these experiments suggest that the flow modifier should be as thin as possible. In this work, the thickness of the thinner modifier is similar to the thickness of the walls of the nozzle. However, it would be desirable to analyze the feasibility of using a flow modifier with an even smaller thickness.

However, the risk, of using a too thin modifier, is that the modifier may break due to temperatures and mechanical stresses associated with normal operational conditions.

The results of this work recommend that the length of the modifier should allow a gap between the edge of each modifier and its corresponding narrow wall of the mold. The length of the gap must be at least a half of the mold thickness.

\section{Acknowledgments}

F. Rivera-Perez thanks to Universidad Autonoma Metropolitana for Ph.D. Grant. R. Hernandez-Santoyo thanks to Consejo Nacional de Ciencia y Tecnologia (CONACYT) for Grant No. 284913. J. Gonzalez-Trejo, C. Real-Ramirez, R. Miranda-Tello, F. Cervantes-de-la-Torre thank the SNI for the distinction granted and the stipend received. The numerical and physical simulations were done in the Laboratorio de Computo CientificoDepartamento de Sistemas at Universidad Autonoma Metropolitana-Azcapotzalco.

\section{REFERENCES}

[1] C.H. Yim , O. Kwon, J. Iron Steel Res. Int. 15, $52-58$ (2008).

[2] B. You, T. Sim, M. Kim, D. Lee, J. Lee, J. Lee, ISIJ Int. 49, 11741183 (2009).

[3] L.F. Zhang, B.G. Thomas, ISIJ Int. 43, 271-291 (2003).

[4] K. Timmel, X. Miao, T. Wondrak, F. Stefani, D. Lucas, S. Eckert, G. Gerbeth, Eur. Phys. J. Spec. Top. 220, 151-166 (2013).

[5] Y.J. Jeon, H.J. Sung, S. Lee, Metall. Mater. Trans. B 41, 121-130 (2010).

[6] V. Singh, S.K. Dash, J.S. Sunitha, S.K. Ajmani, A.K. Das, ISIJ Int. 46, 210-218 (2006).

[7] B.Z. Shen, H.F. Shen, B.C. Liu, ISIJ Int. 47, 427-432 (2007).

[8] B.Z. Shen, H.F. Shen, B.C. Liu, Ironmak Steelmak. 36, 33-38 (2009).

[9] H.F. Shen, B.Z. Shen, B.C. Liu, Steel Res. Int. 78, 531-535 (2007).

[10] R. Miranda, M.A. Barron, J. Barreto, L. Hoyos, J. Gonzalez, ISIJ Int. 45, 1626-1635 (2005).

[11] R.D. Dauby, H. Pierre, AIST Transactions 8, 152-160 (2011).

[12] T.M.J. Kalter, S. Kenjeres, C.R. Kleijn, International Journal of Heat and Fluid Flow 2012).

[13] Y. Chen, L. Zhang, S. Yang, J. Li, JOM 64, 1080-1086 (2012).

[14] M. Kamal, Y. Sahai, ISIJ Int. 46, 1823-1832 (2006).

[15] Y.H. Wu, B. Wiwatanapataphee, Discrete Contin. Dyn. Syst.-Ser. B 8, 695-706 (2007).

[16] F. Rivera-Perez, C. Real-Ramirez, R. Miranda-Tello, R. Hernandez-Santoyo, F. Cervantes-de la Torre, J. Gonzalez-Trejo, Mathematical Problems in Engineering 2014, 12 (2014).

[17] ANSYS Inc., ANSYS 6.3 Theory Guide, ed. ANSYS. Vol. 6.3. 2006, Canonsburg: ANSYS. 